Morphisms of Real Calculi

from a Geometric and

Algebraic Perspective

Axel Tiger Norkvist 



\title{
Morphisms of Real Calculi from a Geometric and Algebraic Perspective
}

\author{
Axel Tiger Norkvist
}

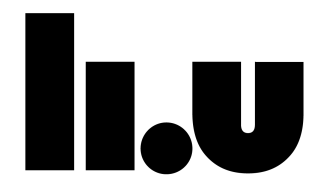

LINKÖPING UNIVERSITY

Department of Mathematics

Linköping, 2021 
$(\mathrm{ccc)E \textrm {EY } - \mathrm { NC }}$ This work is licensed under a Creative Commons AttributionNonCommercial 4.0 International License.

https://creativecommons.org/licenses/by-nc/4.0/

Linköping Studies in Science and Technology. Licenciate Thesis No. 1913

Morphisms of Real Calculi from a Geometric and Algebraic Perspective

Copyright (C) Axel Tiger Norkvist, 2021

Department of Mathematics

Linköping University

SE-581 83 Linköping, Sweden

Email: axel.tiger.norkvist@liu.se

ISSN 0280-7971

ISBN 978-91-7929-616-2

Printed by LiU-Tryck, Linköping, Sweden, 2021

This is a Swedish Licentiate Thesis.

The Licentiate degree comprises 120 ECTS credits of postgraduate studies. 


\section{Abstract}

Noncommutative geometry has over the past four of decades grown into a rich field of study. Novel ideas and concepts are rapidly being developed, and a notable application of the theory outside of pure mathematics is quantum theory. This thesis will focus on a derivation-based approach to noncommutative geometry using the framework of real calculi, which is a rather direct approach to the subject. Due to their direct nature, real calculi are useful when studying classical concepts in Riemannian geometry and how they may be generalized to a noncommutative setting.

This thesis aims to shed light on algebraic aspects of real calculi by introducing a concept of morphisms of real calculi, which enables the study of real calculi on a structural level. In particular, real calculi over matrix algebras are discussed both from an algebraic and a geometric perspective. Morphisms are also interpreted geometrically, giving a way to develop a noncommutative theory of embeddings. As an example, the noncommutative torus is minimally embedded into the noncommutative 3-sphere. 


\section{Sammanfattning}

Ickekommutativ geometri har under de senaste fyra decennierna blivit ett etablerat forskningsområde inom matematiken. Nya idéer och koncept utvecklas i snabb takt, och en viktig fysikalisk tillämpning av teorin är inom kvantteorin. Denna avhandling kommer att fokusera på ett derivationsbaserat tillvägagångssätt inom ickekommutativ geometri där ramverket real calculi används, vilket är ett relativt direkt sätt att studera ämnet på. Eftersom analogin mellan real calculi och klassisk Riemanngeometri är intuitivt klar så är real calculi användbara när man undersöker hur klassiska koncept inom Riemanngeometri kan generaliseras till en ickekommutativ kontext.

Denna avhandling ämnar att klargöra vissa algebraiska aspekter av real calculi genom att introducera morfismer för dessa, vilket möjliggör studiet av real calculi på en strukturell nivå. I synnerhet diskuteras real calculi över matrisalgebror från både ett algebraiskt och ett geometriskt perspektiv. Morfismer tolkas även geometriskt, vilket leder till en ickekommutativ teori för inbäddningar. Som ett exempel blir den ickekommutativa torusen minimalt inbäddad i den ickekommutativa 3 -sfären. 


\section{Acknowledgments}

First and foremost, I would like to thank my main supervisor Joakim Arnlind. For the past two and a half years he has been a great mentor during my graduate education, providing me with expert advice and feedback on my work. Our many discussions have truly enriched my perspectives on many things, and have greatly helped in forming my current views on mathematics as a whole.

Secondly I would like to thank my fellow PhD students at MAI. While deciding to become a $\mathrm{PhD}$ student is certainly not something I regret, it is not a common path to take and sometimes it is reassuring to talk to people who share similar experiences to myself. My fellow $\mathrm{PhD}$ students have always been open to talk and made sure I feel welcome, and even though the pandemic makes meeting in person less frequent I've still found immense value in having friendly peers during my education.

Lastly, I would like to thank my family. The pursuit of a $\mathrm{PhD}$ in mathematics is certainly an unconventional career decision to make, but my family have been nothing but supportive throughout. I count myself lucky to have family members that trust me in my ability to decide my life on my own terms, and whom I know that I can turn to for support in times of need. 


\section{Contents}

Abstract ...................... i

Sammanfattning . . . . . . . . . . . . . . . . iii

Acknowledgments . . . . . . . . . . . . . . . . . . . . . v v

Contents. . . . . . . . . . . . . . . . . . . . vii

\begin{tabular}{|ll|}
\hline 1 & Riemannian geometry and its generalization to noncommutative \\
\hline \hline geometry & 1
\end{tabular}

1.1 Metrics and affine connections. . . . . . . . . . . . . . . . 1

1.2 Generalizing geometry to noncommutative spaces . . . . . . . . . . 2

1.3 Introducing real calculi $\ldots \ldots \ldots \ldots \ldots \ldots$

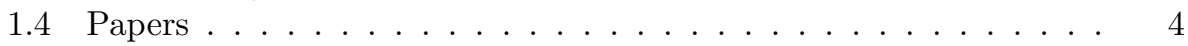

\begin{tabular}{|lll}
\hline 2 & Real calculi and their morphisms & 7
\end{tabular}

2.1 Real metric calculi and affine connections . . . . . . . . . 7

2.2 Real calculus homomorphisms . . . . . . . . . . . . . . . . 9

$2.3 \quad$ Free and projective real calculi $\ldots \ldots \ldots \ldots . \ldots . \ldots . \ldots 12$

$\begin{array}{lll}3 \text { A theory of noncommutative embeddings } & 19\end{array}$

3.1 General embeddings $\ldots \ldots \ldots \ldots \ldots$

3.2 Isometric embeddings $\ldots \ldots \ldots \ldots \ldots \ldots$

4 The noncommutative torus and 3-sphere 25

4.1 The noncommutative torus $\ldots \ldots \ldots \ldots \ldots \ldots$

4.2 The noncommutative 3 -sphere. . . . . . . . . . . . . . . 26

$4.3 \quad$ An embedding of the noncommutative torus . . . . . . . . . . . . . 29

\begin{tabular}{|lll}
5 & Real calculi over matrix algebras & 33
\end{tabular}

5.1 Matrix algebras and classification . . . . . . . . . . . . . . 33

5.2 The 1 -dimensional case . . . . . . . . . . . . . . . . . 35

5.3 Abelian Lie algebras $\ldots \ldots \ldots \ldots$

$\begin{array}{lll}6 \text { Outlook } & 45\end{array}$

\begin{tabular}{ll}
\hline Paper I & 47
\end{tabular}

\begin{tabular}{ll}
\hline Paper II & 67
\end{tabular} 


\section{1 - Riemannian geometry and its generalization to noncommutative geometry}

Since the early 1980's, noncommutative geometry has grown into an important branch of modern mathematics. By adopting a radically different approach to geometry where coordinates are no longer required to commute, a rich field of study has been uncovered, which has various interesting applications both in pure mathematics as well as quantum physics. In this introductory chapter we seek to give a basic intuitive motivation behind noncommutative geometry, and introduce the framework of real calculi which will be used in this thesis to represent noncommutative spaces as a direct generalization of Riemannian manifolds.

\subsection{Metrics and affine connections}

In this section we briefly recall some central concepts from Riemannian geometry which shall be generalized to a noncommutative context in Chapter 2. This section is far from exhaustive, and many aspects that are normally considered with regards to Riemannian geometry (such as curvature) are not discussed below. Indeed, we only describe metrics and affine connections, which are directly relevant to the material covered in the rest of this thesis, and we describe them in a similar way to their generalizations in Chapter 2.

In differential geometry the central object of study is a Riemannian manifold $(\Sigma, g)$, where the smooth manifold $\Sigma$ encodes the differential and topological structure and the metric $g$ describes the geometry of $(\Sigma, g)$ by acting as a scalar product between tangent vectors at each point of $\Sigma$.

Using the module $\operatorname{Vect}(\Sigma) \simeq \operatorname{Der}\left(C^{\infty}(\Sigma)\right)$ of smooth vector fields, the metric $g$ can also be seen as a tensor field, where $g(X, Y)$ is a smooth real-valued function on the manifold for $X, Y \in \operatorname{Vect}(\Sigma)$. In other words, $g$ can be seen as a bilinear map from $\operatorname{Vect}(\Sigma) \times \operatorname{Vect}(\Sigma)$ to $C^{\infty}(\Sigma)$.

Besides the metric, another central concept in differential geometry is affine connections as a way to "connect nearby tangent spaces", making it possible to develop a notion of differentiation of vector fields. More precisely, an affine connection $\nabla: \operatorname{Vect}(\Sigma) \times \operatorname{Vect}(\Sigma) \rightarrow \operatorname{Vect}(\Sigma)$ is a map that satisfies

1. $\nabla_{f X+Y} Z=f \nabla_{X} Z+\nabla_{Y} Z$,

2. $\nabla_{X}(Y+Z)=\nabla_{X} Y+\nabla_{X} Z$,

3. $\nabla_{X}(f Y)=f \nabla_{X} Y+X(f) Y$, 
for $X, Y, Z \in \operatorname{Vect}(\Sigma)$ and $f \in C^{\infty}(\Sigma)$, where $X(f)$ is the directional derivative of $f$ with respect to $X$, thus making condition 3 a variant of the Leibniz condition for derivations.

Generally speaking, there is no canonical affine connection on a differentiable manifold. But on Riemannian manifolds there is a preferred connection, called the Levi-Civita connection, which is the unique connection that is an isometry under parallell transport of tangent vectors and which also has vanishing torsion. In other words, the Levi-Civita connection is the unique affine connection that satisfies

(T) $\nabla_{X} Y-\nabla_{Y} X-[X, Y]=0$ for all $X, Y \in \operatorname{Vect}(\Sigma)$,

(M) $X(g(Y, Z))=g\left(\nabla_{X} Y, Z\right)+g\left(Y, \nabla_{X} Z\right)$ for all $X, Y, Z \in \operatorname{Vect}(\Sigma)$,

which can be summarized into Koszul's classical formula:

$$
\begin{aligned}
2 g\left(\nabla_{X} Y, Z\right)= & X(g(Y, Z))+Y(g(X, Z))-Z(g(X,)) \\
& -g([Y, Z], X)+g([Z, X], Y)+g([X, Y], Z) ;
\end{aligned}
$$

Using invertibility of the metric $g$, the above formula can be used to find $\nabla$ explicitly.

\subsection{Generalizing geometry to noncommutative spaces}

In order to generalize the differential geometry of Riemannian manifolds to a noncommutative context, a radically new perspective needs to be adopted. The key insight we shall use is the following: in the previous section on metrics and affine connections, both the metric $g$ and connection $\nabla$ were purposefully described algebraically, and not in terms of their values at specific points on the manifold. In fact, if we have an algebra $\mathcal{A}$ which can be interpreted as the algebra of smooth functions on a manifold $\Sigma$ and we choose the module $M=\operatorname{Der}(\mathcal{A})$ which can then be interpreted as the module of smooth vector fields on $\Sigma$, we can define metrics and affine connections algebraically in terms of $\mathcal{A}$ and $M$, without explicitly mentioning the manifold. We can take this line of reasoning even further; as long as these definitions make sense for certain classes of algebras and modules, they can be studied even though the algebras may not have a direct connection to classical geometry, which inspired the definitions to begin with.

A natural question to ask when formulating geometry algebraically is which types of algebras correspond to which types of manifolds. An important result, which is the starting point for noncommutative geometry in general, is the GelfandNaimark theorem for commutative $C^{*}$-algebras, i.e., commutative ${ }^{*}$-algebras $\mathcal{A}$ which are also Banach algebras equipped with a norm that satisfies $\left\|x^{*} x\right\|=\|x\|^{2}$ for all $x \in \mathcal{A}$. We state it here as follows.

Theorem 1.2.1 (Gelfand-Naimark). Let $\mathcal{A}$ be a commutative (unital) $C^{*}$-algebra. Then $\mathcal{A}$ is isometrically ${ }^{*}$-isomorphic to $C_{0}(X)$ for some (compact) Hausdorff space $X$. 
While it is straightforward to check that $C_{0}(X)$ is a commutative (unital) $C^{*}$ algebra for any (compact) Hausdorff space (with $C_{0}(X) \simeq C_{0}(Y)$ if $X$ and $Y$ are homeomorphic), the converse is less clear, making the above theorem an important milestone in the theory of operator algebras.

In light of the Gelfand-Naimark theorem, the following kinds of questions become highly interesting: what happens if we consider noncommutative $C^{*}$ algebras? What kind of "geometric objects" do they correspond to? The study of these questions have led to the development of important mathematical concepts; as an example, see cyclic (co)homology developed by Alain Connes in Con85, which can (for instance) be used to study the space of leaves of a foliation, or to find generalizations of the famous Atiyah-Singer index theorem. The resulting branch of mathematics that evolved (called noncommutative geometry) started to become increasingly interesting to theoretical physicists in the late 1990's (cf. SW99]). To this day, noncommutative geometry remains a rich field of study, with many questions and concepts left to explore.

\subsection{Introducing real calculi}

In this thesis we will consider one way of generalizing aspects of geometry to a noncommutative setting using the framework of real calculi. Having been introduced in AW17b, this derivation-based approach is still under development and many questions have yet to be answered; indeed, it is not yet completely clear what role real calculi will play in the future of noncommutative geometry in general. One main advantage it has over other more established frameworks, such as spectral triples (cf. Con94]), is that it is a very direct approach to the subject and it is often straightforward how concepts "should be" defined, making it easy to have educated guesses on how definitions and results from differential geometry can be generalized to real calculi. Worth noting, however, is that real calculi is not a radically different approach, and several other approaches to noncommutative geometry that share some conceptual similarities to real calculi have been taken before (see e.g. [LM87, FGR99, BM11, Ros13]). In particular it can be noted derivation-based approaches to noncommutative geometry, pioneered by Michel Dubois-Violette et al. in the late 1980's and throughout the 1990's (for instance, see [DV88, DVKM90]), are well-known and an established part of the field.

Definition 1.3.1 (Real calculus). Let $\mathcal{A}$ be a unital *-algebra, and let $\mathfrak{g}_{D}$ denote a real Lie algebra together with a faithful representation $D: \mathfrak{g} \rightarrow \operatorname{Der}(\mathcal{A})$ that maps each $\partial \in \mathfrak{g}$ to a hermitian derivation. Moreover, let $M$ be a right $\mathcal{A}$-module and let $\varphi: \mathfrak{g} \rightarrow M$ be a $\mathbb{R}$-linear map such that $M$ is generated from $\varphi(\mathfrak{g})$. Then $C_{\mathcal{A}}=\left(\mathcal{A}, \mathfrak{g}_{D}, M, \varphi\right)$ is called a real calculus over $\mathcal{A}$.

As a convention, we write $\partial(a)$ instead of the more cumbersome $D(\partial)(a)$ for an element $\partial \in \mathfrak{g}$ acting on an element $a \in \mathcal{A}$ as a derivation, as long as there is no risk of confusion. Moreover, if $\mathfrak{g} \subseteq \operatorname{Der}(\mathcal{A})$ and the representation $D$ is left unspecified, then $D$ is implicitly assumed to be the identity map on $\mathfrak{g}$. An important idea in noncommutative geometry is that it is a generalization of classical geometry, 
and therefore it is helpful to see exactly how a smooth manifold can be realized through the use of real calculi.

Example 1. Let $\Sigma$ be a compact smooth manifold. If we let $\mathcal{A}=C^{\infty}(\Sigma)$, $\mathfrak{g}=\operatorname{Der}(\mathcal{A})$ (with $D$ simply being the identity mapping on $\mathfrak{g}$ ), $M$ be the module of smooth vector fields on $\Sigma$, and finally let $\varphi: \mathfrak{g} \rightarrow M$ be the natural correspondence between derivations and smooth vector fields, then the real calculus $C_{\mathcal{A}}=\left(\mathcal{A}, \mathfrak{g}_{D}, M, \varphi\right)$ can be used to represent the manifold $\Sigma$.

It is worth noting a few details in particular. Firstly, since real calculi are defined over general *-algebras without requiring them to be normed, the condition that the *-algebra is unital does not imply that only compact (noncommutative) spaces can be studied in the framework; for instance, consider [AL20], where a real calculus over the noncommutative cylinder is described.

Secondly, in Example 1. where $\mathcal{A}=C^{\infty}(\Sigma)$ is commutative, the natural correspondence between derivations and vector fields implies that the entire smooth structure of $\Sigma$ can be recovered directly from $\left(\mathcal{A}, \mathfrak{g}_{D}\right)$, making $M$ and $\varphi$ somewhat redundant in the structure $C_{\mathcal{A}}=\left(\mathcal{A}, \mathfrak{g}_{D}, M, \varphi\right)$.

However, when $\mathcal{A}$ is noncommutative we generally have that the set $\operatorname{Der}(\mathcal{A})$ does not have a module structure over $\mathcal{A}$, since it is not true in general that $a \partial \in \operatorname{Der}(\mathcal{A})$ for every $a \in \mathcal{A}, \partial \in \operatorname{Der}(\mathcal{A})$. Therefore, if we wish to investigate noncommutative spaces with a derivation-based approach and introduce a metric to describe their "geometry", it becomes natural to include an $\mathcal{A}$-module $M$ on which the metric can be realized as a nondegenerate bilinear map; since $M$ is associated with the notion of vector fields in classical geometry, an explicit description of the relationship between derivations in $\mathfrak{g}$ and "vector fields" in $M$ is warranted. Hence, both $M$ and $\varphi: \mathfrak{g} \rightarrow M$ contribute to the overall "differential" structure of $C_{\mathcal{A}}=\left(\mathcal{A}, \mathfrak{g}_{D}, M, \varphi\right)$ when $\mathcal{A}$ is noncommutative.

Finally, real calculi are essentially algebraic structures, implying that this approach to noncommutative geometry is algebraic in nature rather than analytic. This has the direct consequence that real calculi as a framework are less powerful than spectral triples, where the power of functional analysis is used to develop sophisticated tools to gain deep insights into noncommutative spaces. However, real calculi are relatively simple to work with, and they are a good testing ground for seeing how concepts in classical Riemannian geometry can (and can not) be generalized.

\subsection{Papers}

The following papers are included, which elaborate on the topics presented in the main thesis.

\section{Paper I - Noncommutative minimal embeddings and morphisms of pseudo- Riemannian calculi}

Abstract. In analogy with classical submanifold theory, we introduce morphisms of real metric calculi together with noncommutative embeddings. We show that basic 
concepts, such as the second fundamental form and the Weingarten map, translate into the noncommutative setting and, in particular, we prove a noncommutative analogue of Gauss' equations for the curvature of a submanifold. Moreover, the mean curvature of an embedding is readily introduced, giving a natural definition of a noncommutative minimal embedding, and we illustrate the novel concepts by considering the noncommutative torus as a minimal surface in the noncommutative 3 -sphere.

\section{Paper II - Projective real calculi over matrix algebras}

Abstract. In analogy with the geometric situation, we study real calculi over projective modules and describe how they are related to free real calculi using real calculus homomorphisms. Moreover, we study real calculi over matrix algebras and discuss several aspects of the classification problem for real calculi in this case. We also use matrix algebras to give concrete examples of real calculi where the module is projective, and how this affects the existence of a Levi-Civita connection. 



\section{2 - Real calculi and their morphisms}

Real calculi as introduced in Definition 1.3.1 are useful when studying potential generalizations of classical concepts in Riemannian geometry since there are clear analogues between a real calculus over a noncommutative algebra and a differential structure on a manifold. As described in Chapter 1, if $C_{\mathcal{A}}=\left(\mathcal{A}, \mathfrak{g}_{D}, M, \varphi\right)$ is a real calculus then we think of $\mathcal{A}$ as the algebra of functions, $\mathfrak{g}$ as the Lie algebra of derivations and $M$ as the module of vector fields over a "manifold". As it stands, $C_{\mathcal{A}}$ contains all the topological and differential structure of the manifold, but in order to study Riemannian geometry in this framework it becomes important to define metrics and affine connections. In this chapter we shall also develop the central notion of this thesis, which is real calculus homomorphisms. These morphisms can be studied both from an algebraic and a geometric perspective, leading to many insights regarding the structure of a real calculus. Later on in this chapter we shall also describe free and projective real calculi, which are the most interesting types of real calculi from a geometric perspective, and we describe how morphisms of real calculi give us a way to view projective real calculi as "projections" of free real calculi.

\subsection{Real metric calculi and affine connections}

In the context of real calculi, it is natural to define the metric as a nondegenerate bilinear form on the module $M$ of vector fields; we make this notion precise in the following.

Definition 2.1.1. Let $\mathcal{A}$ be a ${ }^{*}$-algebra and let $M$ be a right $\mathcal{A}$-module. $\mathrm{A}$ hermitian form on $M$ is a map $h: M \times M \rightarrow \mathcal{A}$ with the following properties:

$$
\begin{aligned}
& \text { h1. } h\left(m_{1}, m_{2}+m_{3}\right)=h\left(m_{1}, m_{2}\right)+h\left(m_{1}, m_{3}\right) \\
& \text { h2. } h\left(m_{1}, m_{2} a\right)=h\left(m_{1}, m_{2}\right) a \\
& \text { h3. } h\left(m_{1}, m_{2}\right)=h\left(m_{2}, m_{1}\right)^{*}
\end{aligned}
$$

for every $m_{1}, m_{2}, m_{3} \in M$ and $a \in \mathcal{A}$. Moreover, if $h\left(m_{1}, m_{2}\right)=0$ for every $m_{2} \in M$ implies that $m_{1}=0$ then $h$ is said to be nondegenerate, and in this case we say that $h$ is a metric on $M$. The pair $(M, h)$ is called a (right) hermitian $\mathcal{A}$ module, and if $h$ is a metric on $M$ we say that $(M, h)$ is a (right) metric $\mathcal{A}$-module. Finally, we say that $h$ is invertible if the map $\hat{h}: M \rightarrow M^{*}$, defined by the formula $\hat{h}(m)(n)=h(m, n)$, is invertible.

Definition 2.1.2 (Real metric calculus). Let $C_{\mathcal{A}}=\left(\mathcal{A}, \mathfrak{g}_{D}, M, \varphi\right)$ be a real calculus over $\mathcal{A}$ and let $(M, h)$ be a (right) metric $\mathcal{A}$-module. If

$$
h\left(\varphi\left(\partial_{1}\right), \varphi\left(\partial_{2}\right)\right)^{*}=h\left(\varphi\left(\partial_{1}\right), \varphi\left(\partial_{2}\right)\right)
$$


for every $\partial_{1}, \partial_{2} \in \mathfrak{g}$ then the pair $\left(C_{\mathcal{A}}, h\right)$ is called a real metric calculus.

The hermeticity conditions in the above definition can be seen as a way to impose a "real" structure on the calculus.

Example 2. As in Example 1, let $\Sigma$ be a compact smooth manifold and let $C_{\mathcal{A}}=$ $\left(\mathcal{A}, \mathfrak{g}_{D}, M, \varphi\right)$ be the real calculus representing $\Sigma$ (i.e., $\mathcal{A}=C^{\infty}(\Sigma), \mathfrak{g}=\operatorname{Der}(\mathcal{A})$ and $M=\operatorname{Vect}(\Sigma))$. If $g$ is a Riemannian metric on $\operatorname{Vect}(\Sigma)$, then $\left(C_{\mathcal{A}}, g\right)$ is a real metric calculus representing the Riemannian manifold $(\Sigma, g)$.

Let $(\Sigma, g)$ be a (pseudo-)Riemannian manifold. By the fundamental theorem of Riemannian geometry there is a unique connection $\nabla$ that preserves the metric $g$ and is torsion-free (the Levi-Civita connection), and in order to understand to what extent this can be generalized we need to define the notion of a connection in the context of real calculi. The main difference between affine connections in this context as compared with the classical situation is that the connection $\nabla$ can be seen as differentiation of vector fields with respect to derivations rather than vector fields, since real calculi does not assume a one-to-one correspondence between vector fields and derivations.

Definition 2.1.3. Let $C_{\mathcal{A}}=\left(\mathcal{A}, \mathfrak{g}_{D}, M, \varphi\right)$ be a real calculus over $\mathcal{A}$. An affine connection on $(M, \mathfrak{g})$ is a map $\nabla: \mathfrak{g} \times M \rightarrow M$ satisfying

1. $\nabla_{\partial}(m+n)=\nabla_{\partial} m+\nabla_{\partial} n$

2. $\nabla_{\lambda \partial+\partial^{\prime}} m=\lambda \nabla_{\partial} m+\nabla_{\partial^{\prime}} m$

3. $\nabla_{\partial}(m a)=\left(\nabla_{\partial} m\right) a+m \partial(a)$

for $m, n \in M, \partial, \partial^{\prime} \in \mathfrak{g}, a \in \mathcal{A}$ and $\lambda \in \mathbb{R}$.

The third condition in the above condition can be seen as a Leibniz condition, supporting the view of $\nabla_{\partial} m$ as a "derivative" of $m$ with respect to $\partial$. Much like real metric calculi, a real structure is imposed on the calculus when discussing connections by adding hermiticity conditions for the connection to satisfy with respect to the metric.

Definition 2.1.4 (Real connection calculus). Let $\left(C_{\mathcal{A}}, h\right)$ be a real metric calculus and let $\nabla$ be an affine connection on $(M, \mathfrak{g})$. Then $\left(C_{\mathcal{A}}, h, \nabla\right)$ is called a real connection calculus if

$$
h\left(\nabla_{\partial} \varphi\left(\partial_{1}\right), \varphi\left(\partial_{2}\right)\right)=h\left(\nabla_{\partial} \varphi\left(\partial_{1}\right), \varphi\left(\partial_{2}\right)\right)^{*}
$$

for every $\partial, \partial_{1}, \partial_{2} \in \mathfrak{g}$.

Definition 2.1.5 (Pseudo-Riemannian calculus). Let $\left(C_{\mathcal{A}}, h, \nabla\right)$ be a real connection calculus. We say that $\left(C_{\mathcal{A}}, h, \nabla\right)$ is metric if

$$
\partial\left(h\left(m_{1}, m_{2}\right)\right)=h\left(\nabla_{\partial} m_{1}, m_{2}\right)+h\left(m_{1}, \nabla_{\partial} m_{2}\right)
$$

for every $\partial \in \mathfrak{g}$ and $m_{1}, m_{2} \in M$, and torsion-free if

$$
\nabla_{\partial_{1}} \varphi\left(\partial_{2}\right)-\nabla_{\partial_{2}} \varphi\left(\partial_{1}\right)-\varphi\left(\left[\partial_{1}, \partial_{2}\right]\right)=0
$$

for every $\partial_{1}, \partial_{2} \in \mathfrak{g}$. A metric and torsion-free real connection calculus is called a pseudo-Riemannian calculus. 
A connection fulfilling the conditions for a pseudo-Riemannian connection is called a Levi-Civita connection. Unlike classical Riemannian geometry, in the quite general setup of real metric calculi the existence of a Levi-Civita connection can not be guaranteed. However, it is unique if it exists.

Theorem 2.1.6 (Levi-Civita connection, AW17b]). Let $\left(C_{\mathcal{A}}, h\right)$ be a real metric calculus. Then there exists at most one affine connection $\nabla$ such that $\left(C_{\mathcal{A}}, h, \nabla\right)$ is a pseudo-Riemannian calculus).

In order to check whether a given connection is indeed the Levi-Civita connection, it is often practical to use the classical Koszul formula. Moreover, this formula can sometimes be used to define the Levi-Civita connection if it exists. We state it as follows:

Proposition 2.1.7 (Koszul's formula, AW17b]). Let $\left(C_{\mathcal{A}}, h, \nabla\right)$ be a pseudoRiemannian calculus and assume that $\partial_{1}, \partial_{2}, \partial_{3} \in \mathfrak{g}$. Then it holds that

$$
\begin{aligned}
2 h\left(\nabla_{1} e_{2}, e_{3}\right)= & \partial_{1} h\left(e_{2}, e_{3}\right)+\partial_{2} h\left(e_{1}, e_{3}\right)-\partial_{3} h\left(e_{1}, e_{2}\right) \\
& -h\left(e_{1}, \varphi\left(\left[\partial_{2}, \partial_{3}\right]\right)\right)+h\left(e_{2}, \varphi\left(\left[\partial_{3}, \partial_{1}\right]\right)\right)+h\left(e_{3}, \varphi\left(\left[\partial_{1}, \partial_{2}\right]\right)\right),
\end{aligned}
$$

where $\nabla_{i}=\nabla_{\partial_{i}}$ and $e_{i}=\varphi\left(\partial_{i}\right)$ for $i=1,2,3$. Conversely, if $\left(C_{\mathcal{A}}, h\right)$ is a real metric calculus and $\nabla$ is a connection satisfying Koszul's formula 2.1) for every $\partial_{1}, \partial_{2}, \partial_{3} \in \mathfrak{g}$, then $\left(C_{\mathcal{A}}, h, \nabla\right)$ is a pseudo-Riemannian calculus.

Later on in this chapter we shall examine projective and free real calculi, which can be viewed as corresponding to the classical concepts of differentiable and parallelizable manifolds. But before doing that we shall introduce the concept of morphisms of real calculi, which will enable us to give a way to tell when two real calculi are essentially the same structure viewed from (possibly) different perspectives.

\subsection{Real calculus homomorphisms}

Real calculi are algebraic structures and in order to study them as such it becomes necessary to define a notion of a homomorphism of real calculi. Throughout the rest of this thesis, the majority of the theory presented is connected to this central concept in some way. Real calculus homomorphisms are necessary when determining whether two given real calculi are essentially the same structure (i.e., whether they are isomorphic), but they were originally developed in ATN21 as a geometric tool to discuss embeddings in a noncommutative context; this perspective shall be treated in more detail in Chapters 3 and 4

Definition 2.2.1. Let $C_{\mathcal{A}}=\left(\mathcal{A}, \mathfrak{g}_{D}, M, \varphi\right)$ and $C_{\mathcal{A}^{\prime}}=\left(\mathcal{A}^{\prime}, \mathfrak{g}_{D^{\prime}}^{\prime}, M^{\prime}, \varphi^{\prime}\right)$ be real calculi and let $\phi: \mathcal{A} \rightarrow \mathcal{A}^{\prime}$ be a ${ }^{*}$-algebra homomorphism. The Lie algebra homomorphism $\psi: \mathfrak{g}^{\prime} \rightarrow \mathfrak{g}$ is said to be compatible with $\phi$ if it satisfies the condition

$$
\phi(\psi(\partial)(a))=\partial(\phi(a))
$$


for every $\partial \in \mathfrak{g}^{\prime}$. If $\psi$ is compatible with $\phi$, then we let $\Psi=\varphi \circ \psi$ and we let $M_{\Psi}$ denote the submodule of $M$ generated by $\Psi\left(\mathfrak{g}^{\prime}\right)$. Furthermore, if the map $\hat{\psi}: M_{\Psi} \rightarrow M^{\prime}$ satisfies the conditions

1. $\hat{\psi}\left(m_{1}+m_{2}\right)=\hat{\psi}\left(m_{1}\right)+\hat{\psi}\left(m_{2}\right)$ for all $m_{1}, m_{2} \in M_{\Psi}$,

2. $\hat{\psi}(m a)=\hat{\psi}(m) \phi(a)$ for all $m \in M_{\Psi}, a \in \mathcal{A}$,

3. $\hat{\psi}(\Psi(\partial))=\varphi^{\prime}(\partial)$ for all $\partial \in \mathfrak{g}^{\prime}$,

then $\hat{\psi}$ is said to be compatible with $\phi$ and $\psi$, and we say that $(\phi, \psi, \hat{\psi})$ is a real calculus homomorphism from $C_{\mathcal{A}}$ to $C_{\mathcal{A}^{\prime}}$; if $\phi$ and $\psi$ are isomorphisms and $\hat{\psi}$ is a bijection, then $(\phi, \psi, \hat{\psi}): C_{\mathcal{A}} \rightarrow C_{\mathcal{A}^{\prime}}$ is called a real calculus isomorphism.

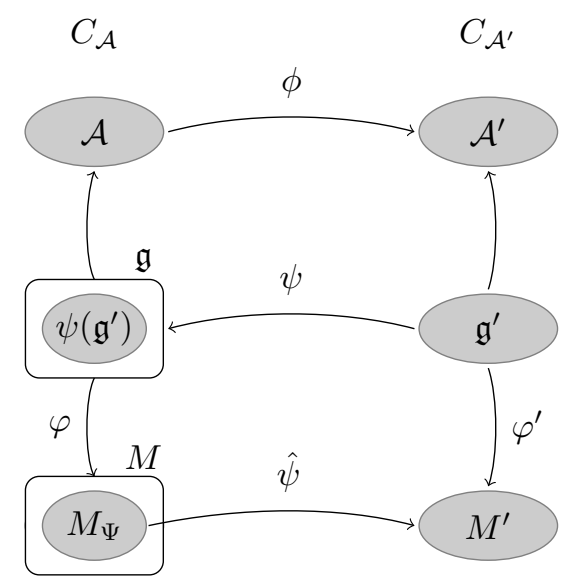

Figure 2.1: Illustration of a real calculus homomorphism.

Let us describe the above definition in terms of Figure 2.1. Compatibility between $\phi$ and $\psi$ corresponds to $\phi$ and $\psi$ "commuting" in the upper half of the figure (although technically speaking, the upper half of Figure 2.1 is not a commutative diagram), whereas Condition 3 corresponds to the lower half of the figure (which involves $\psi, \hat{\psi}, \varphi$ and $\varphi^{\prime}$ ) being a commutative diagram.

One thing that may seem unnatural at first glance is that the map $\psi$ goes in the "wrong" direction, mapping elements in $\mathfrak{g}^{\prime}$ to elements in $\mathfrak{g}$ instead of the other way around. If we view real calculus homomorphisms purely algebraically there is seemingly no reason why $\psi$ maps in this direction, but if we adopt a geometric perspective matters become more clear. As we shall describe in more detail in Chapter 3, real calculus homomorphisms are naturally connected to the concept of embeddings, and by this interpretation the set $M_{\Psi}$ can be viewed as the vector fields on the ambient manifold that are tangent to the submanifold, with the map $\hat{\psi}$ being the restriction of these to the submanifold; the map $\psi$ can be viewed as the tangent map of an embedding. 
In the next chapter we shall develop a notion of isometric embeddings for real calculi, and to this end we shall need a concept of morphisms between real metric calculi. Since real metric calculi have more structure to take into account than real calculi it is necessary to have additional compatibility conditions for metrics when defining morphisms of real metric calculi.

Definition 2.2.2. Let $\left(C_{\mathcal{A}}, h\right)$ and $\left(C_{\mathcal{A}^{\prime}}, h^{\prime}\right)$ be real metric calculi and let $(\phi, \psi, \hat{\psi})$ : $C_{\mathcal{A}} \rightarrow C_{\mathcal{A}^{\prime}}$ be a real calculus homomorphism. If, additionally,

$$
h^{\prime}\left(\varphi^{\prime}\left(\partial_{1}\right), \varphi^{\prime}\left(\partial_{2}\right)\right)=\phi\left(h\left(\Psi\left(\partial_{1}\right), \Psi\left(\partial_{2}\right)\right)\right)
$$

for every $\partial_{1}, \partial_{2} \in \mathfrak{g}^{\prime}$, then $(\phi, \psi, \hat{\psi}):\left(C_{\mathcal{A}}, h\right) \rightarrow\left(C_{\mathcal{A}^{\prime}}, h^{\prime}\right)$ is called a real metric calculus homomorphism.

While the definition of real calculus homomorphisms is motivated by the geometric concept of embeddings, morphisms are essential for classifying real calculi as algebraic structures as well. For the purpose of understanding real calculus homomorphisms from an algebraic perspective, we state a few basic facts about real calculus homomorphisms. We begin by noting that the composition of real calculus homomorphisms is, again, a real calculus homomorphism.

Proposition 2.2.3. Let $C_{\mathcal{A}}, C_{\mathcal{A}^{\prime}}$ and $C_{\mathcal{A}^{\prime \prime}}$ be real calculi and assume that

$$
(\phi, \psi, \hat{\psi}): C_{\mathcal{A}} \rightarrow C_{\mathcal{A}^{\prime}} \text { and }\left(\phi^{\prime}, \psi^{\prime}, \hat{\psi}^{\prime}\right): C_{\mathcal{A}^{\prime}} \rightarrow C_{\mathcal{A}^{\prime \prime}}
$$

are real calculus homomorphisms. Then the composition

$$
\left(\phi^{\prime}, \psi^{\prime}, \hat{\psi}^{\prime}\right) \circ(\phi, \psi, \hat{\psi})=\left(\phi^{\prime} \circ \phi, \psi \circ \psi^{\prime}, \hat{\psi}^{\prime} \circ \hat{\psi}\right): C_{\mathcal{A}} \rightarrow C_{\mathcal{A}^{\prime \prime}}
$$

is a real calculus homomorphism.

A consequence of the compatibility conditions between $\phi, \psi$ and $\psi$ is that the degrees of freedom for the maps $\psi$ and $\hat{\psi}$ decrease once $\phi$ is chosen. Moreover, if $\phi$ and $\psi$ are compatible, then there is at most one map $\hat{\psi}$ such that $(\phi, \psi, \hat{\psi})$ is a real calculus homomorphism. We state this as follows.

Proposition 2.2.4. If $(\phi, \psi, \hat{\psi})$ and $\left(\phi, \psi, \hat{\psi}^{\prime}\right)$ are real calculus homomorphisms from $C_{\mathcal{A}}$ to $C_{\mathcal{A}^{\prime}}$ then $\hat{\psi}=\hat{\psi}^{\prime}$.

Furthermore, if we assume that $\phi$ is an isomorphism, then $\psi$ is determined uniquely by $\phi$.

Proposition 2.2.5. If $(\phi, \psi, \hat{\psi}): C_{\mathcal{A}} \rightarrow C_{\mathcal{A}^{\prime}}$ is a real calculus homomorphism such that $\phi$ is an isomorphism, then $\psi$ is a Lie algebra isomorphism with

$$
D(\psi(\partial))=\phi^{-1} \circ D^{\prime}(\partial) \circ \phi
$$

for $\partial \in \mathfrak{g}^{\prime}$. 
From the above propositions it becomes clear that for any ${ }^{*}$-algebra isomorphism $\phi$ there can be at most one real calculus homomorphism of the form $(\phi, \psi, \hat{\psi})$.

In general, if $C_{\mathcal{A}}=\left(\mathcal{A}, \mathfrak{g}_{D}, M, \varphi\right)$ and $C_{\mathcal{A}^{\prime}}=\left(\mathcal{A}^{\prime}, \mathfrak{g}_{D^{\prime}}^{\prime}, M^{\prime}, \varphi^{\prime}\right)$ are real calculi and $(\phi, \psi, \hat{\psi}): C_{\mathcal{A}} \rightarrow C_{\mathcal{A}^{\prime}}$ is a real calculus homomorphism, then there is a natural $\mathcal{A}$-module structure on $M^{\prime}$ given by

$$
m^{\prime} \cdot a=m^{\prime} \phi(a), \quad m^{\prime} \in M^{\prime}, a \in \mathcal{A} .
$$

In this sense, the right $\mathcal{A}$-modules $M$ and $M^{\prime}$ are isomorphic when $(\phi, \psi, \hat{\psi})$ is an isomorphism, as expected.

Proposition 2.2.6. If $(\phi, \psi, \hat{\psi}): C_{\mathcal{A}} \rightarrow C_{\mathcal{A}^{\prime}}$ is a real calculus isomorphism, then $M=M_{\Psi} \simeq M^{\prime}$.

Given two isomorphic real calculi

$$
C_{\mathcal{A}}=\left(\mathcal{A}, \mathfrak{g}_{D}, M, \varphi\right) \quad \text { and } \quad C_{\mathcal{A}^{\prime}}=\left(\mathcal{A}^{\prime}, \mathfrak{g}_{D^{\prime}}^{\prime}, M^{\prime}, \varphi^{\prime}\right),
$$

the above discussion implies that $\mathcal{A} \simeq \mathcal{A}^{\prime}, \mathfrak{g} \simeq \mathfrak{g}^{\prime}$ and $M \simeq M^{\prime}$. Thus, the classification problem for real calculi boils down to the study of how the maps $D$ and $\varphi$ affect the overall structure of a real calculus $C_{\mathcal{A}}=\left(\mathcal{A}, \mathfrak{g}_{D}, M, \varphi\right)$, where $\mathcal{A}$, $\mathfrak{g}$ and $M$ are fixed. We shall return to the classification problem in Chapter 5 , where real calculi over $\mathcal{A}=\operatorname{Mat}_{N}(\mathbb{C})$ are studied.

\subsection{Free and projective real calculi}

From the classical Serre-Swan theorem we get the natural connection between vector bundles on compact spaces and projective modules over commutative rings, and this notion motivates the study of projective modules over noncommutative rings in noncommutative geometry. Therefore, real calculi where the module is projective are of special interest. Before discussing general real calculi over projective modules, we begin by examining an especially well-behaved class of real calculi (called free real calculi) closely related to the concept of parallelizable manifolds in classical geometry.

\section{Free real calculi}

We begin by defining the concept of a free real calculus. An important nuance is that we not only require the module $M$ to be free, but we also require that the map $\varphi$ maps bases of $\mathfrak{g}$ onto bases of $M$. In this case, $\varphi$ can be thought of as a "parallelization" of the (noncommutative) manifold represented by a free real calculus.

Definition 2.3.1. A real calculus $C_{\mathcal{A}}=\left(\mathcal{A}, \mathfrak{g}_{D}, M, \varphi\right)$ is called free if there exists a basis $\partial_{1}, \ldots, \partial_{n}$ such that $\varphi\left(\partial_{1}\right), \ldots, \varphi\left(\partial_{2}\right)$ is a basis of $M$ as a (right) $\mathcal{A}$-module.

If $C_{\mathcal{A}}=\left(\mathcal{A}, \mathfrak{g}_{D}, M, \varphi\right)$ is a free real calculus, then it is straightforward to show that $\left\{\varphi\left(\partial_{i}\right)\right\}_{1}^{n}$ is a basis of $M$ for every basis $\left\{\partial_{i}\right\}_{1}^{n}$ of $\mathfrak{g}$. This fact can be used to prove the following lemma, which is useful when classifying free real calculi. 
Lemma 2.3.2. Let $C_{\mathcal{A}}=\left(\mathcal{A}, \mathfrak{g}_{D}, M, \varphi\right)$ and $C_{\mathcal{A}^{\prime}}=\left(\mathcal{A}^{\prime}, \mathfrak{g}_{D^{\prime}}^{\prime}, M^{\prime}, \varphi^{\prime}\right)$ be free real calculi, and assume that there are isomorphisms $\phi: \mathcal{A} \rightarrow \mathcal{A}^{\prime}$ and $\psi: \mathfrak{g}^{\prime} \rightarrow \mathfrak{g}$ that are compatible in the sense that

$$
\partial^{\prime}(\phi(a))=\phi\left(\psi\left(\partial^{\prime}\right)(a)\right)
$$

for every $\partial^{\prime} \in \mathfrak{g}^{\prime}$ and $a \in \mathcal{A}$. Then $C_{\mathcal{A}} \simeq C_{\mathcal{A}^{\prime}}$.

Although not explicitly stated in the definition, if $C_{\mathcal{A}}$ is free then the map $\varphi$ is by necessity injective. We state this as a lemma.

Lemma 2.3.3. If $C_{\mathcal{A}}=\left(\mathcal{A}, \mathfrak{g}_{D}, M, \varphi\right)$ is a free real calculus, then $\varphi$ is injective.

Proof. Let $\left\{\partial_{1}, \ldots, \partial_{k}\right\}$ be any basis of $\mathfrak{g}$, and suppose that $\varphi(\partial)=0$ for $\partial \in \mathfrak{g}$. Since $\mathfrak{g}$ is a real Lie algebra it follows that there are unique real coefficients $a^{i}$ such that $\partial=a^{i} \partial_{i}$. To prove that $\partial=0$, we use the fact that the basis $\left\{\partial_{1}, \ldots, \partial_{k}\right\}$ of $\mathfrak{g}$ induces a basis $\left\{\varphi\left(\partial_{1}\right), \ldots, \varphi\left(\partial_{k}\right)\right\}$ of $M$ to deal with the coefficients $a^{i}$; since $\left\{\varphi\left(\partial_{1}\right), \ldots, \varphi\left(\partial_{k}\right)\right\}$ is a basis of $M$, it follows that

$$
0=\varphi(\partial)=\varphi\left(a^{i} \partial_{i}\right)=\varphi\left(\partial_{i}\right) a^{i}
$$

implies that $a^{1}=a^{2}=\ldots=a^{k}=0$. Thus $\partial=0$ and the lemma follows.

Corollary 2.3.4. Let $C_{\mathcal{A}}=\left(\mathcal{A}, \mathfrak{g}_{D}, \mathcal{A}^{n}, \varphi\right)$ be a free real calculus, and suppose that $\mathcal{A}$ has an invariant basis number. Then $\operatorname{dim} \mathfrak{g}=n$.

Proof. Since $\mathcal{A}$ has invariant basis number and $\mathcal{A}^{n}$ has a basis consisting of $n$ elements, it follows that every basis of $\mathcal{A}^{n}$ consists of $n$ elements. Let $\left\{\partial_{1}, \ldots, \partial_{k}\right\}$ be a basis of $\mathfrak{g}$. Then, by Lemma 2.3.3, $\varphi$ is injective and thus $\left|\left\{\varphi\left(\partial_{1}\right), \ldots, \varphi\left(\partial_{k}\right)\right\}\right|=k$ as well. Since $\left\{\varphi\left(\partial_{1}\right), \ldots, \varphi\left(\partial_{k}\right)\right\}$ is a basis it follows that $k=\left|\left\{\varphi\left(\partial_{1}\right), \ldots, \varphi\left(\partial_{k}\right)\right\}\right|=$ $n$, completing the proof.

When dealing with a free real calculus $C_{\mathcal{A}}=\left(\mathcal{A}, \mathfrak{g}_{D}, M, \varphi\right)$ it is straightforward to define metrics $h$ and affine connections $\nabla$ by using a basis of $M$ generated from a basis of $\mathfrak{g}$ by $\varphi$. Moreover, if a metric $h$ is chosen such that $\left(C_{\mathcal{A}}, h\right)$ is a real metric calculus and such that $h$ is invertible, then it is possible to use the invertibility of the metric together with Koszul's formula in order to define a LeviCivita connection $\nabla$ such that $\left(C_{\mathcal{A}}, h, \nabla\right)$ is pseudo-Riemannian. As such, real metric calculi of this kind are of special interest from a geometric perspective.

Definition 2.3.5. A real metric calculus $\left(C_{\mathcal{A}}, h\right)$ is called free if $C_{\mathcal{A}}$ is free and $h$ is invertible.

Proposition 2.3.6. Let $\left(C_{\mathcal{A}}, h\right)$ be a free real metric calculus. Then there exists a unique affine connection $\nabla$ such that $\left(C_{\mathcal{A}}, h, \nabla\right)$ is a pseudo-Riemannian calculus.

For free real metric calculi it is possible to define the Levi-Civita connection explicitly in terms of its Christoffel symbols. We shall outline this process below, but first we briefly clarify the use of the Einstein summation convention used throughout this text. In differential geometry it is customary to interpret any 
expression of the form $x_{i} y^{i}$ or $x^{i} y_{i}$ as a sum over the index $i$, i.e., unless otherwise stated

$$
x_{i} y^{i}=\sum_{i} x_{i} y^{i} \quad \text { and } \quad x^{i} y_{i}=\sum_{i} x^{i} y_{i}
$$

This convention is used for multiple indices as well; as long as an index is repeated in pairs of upper and lower indices for coefficients, that index is to be interpreted as a summation index. For instance,

$$
x_{i} y_{j}^{i} z^{j}=\sum_{i, j} x_{i} y_{j}^{i} z^{j}
$$

unless otherwise stated since the indices $i$ and $j$ appear in pairs of upper and lower indices.

Given a free real metric calculus $\left(C_{\mathcal{A}}, h\right)$ and a basis $\partial_{1}, \ldots, \partial_{n}$ of $\mathfrak{g}$, we write

$$
e_{a}=\varphi\left(\partial_{a}\right) \quad h_{a b}=h\left(e_{a}, e_{b}\right) \quad\left[\partial_{a}, \partial_{b}\right]=f_{a b}^{c} \partial_{c}
$$

with the structure constants $f_{a b}^{c} \in \mathbb{R}$, giving $h\left(e_{a}, \varphi\left(\left[\partial_{b}, \partial_{c}\right]\right)\right)=h_{a r} f_{b c}^{r}$. Since $\hat{h}$ is invertible and $\left\{e_{1}, \ldots, e_{n}\right\}$ is a basis of $M$, it follows that there exist elements $h^{a b} \in$ $\mathcal{A}$ such that $\hat{h}^{-1}\left(\hat{e}^{a}\right)=e_{b} h^{b a}$ for the basis $\left\{\hat{e}^{1}, \ldots, \hat{e}^{n}\right\}$ of $M^{*}$ dual to $\left\{e_{1}, \ldots, e_{n}\right\}$ (i.e., $\left.\hat{e}^{a}\left(e_{b}\right)=\delta_{b}^{a} \mathbb{1}\right)$.

Proposition 2.3.7. The coefficients $h^{a b}$ of the inverse metric satisfy the identities

$$
\left(h^{a b}\right)^{*}=h^{b a} \quad \text { and } \quad h^{a b} h_{b c}=h_{c b} h^{b a}=\delta_{c}^{a} \mathbb{1} .
$$

Proof. the first identity follows directly from

$$
h^{a b}=\hat{e}^{a}\left(\hat{h}^{-1}\left(\hat{e}^{b}\right)\right)=h\left(\hat{h}^{-1}\left(\hat{e}^{a}\right), \hat{h}^{-1}\left(\hat{e}^{b}\right)\right),
$$

and the last identity is most easily seen by noting that the set $\left\{\tilde{e}_{1}, \ldots, \tilde{e}_{n}\right\}$, where $\tilde{e}_{a}:=\hat{h}^{-1}\left(\hat{e}^{a}\right)=e_{i} h^{i a}$, is a basis of $M$. This is a direct consequence of a one-to-one correspondence between linear combinations $\tilde{e}_{a} x^{a}$ in $M$ and linear combinations $x_{a} \hat{e}^{a}$ in $M^{*}$, with $x_{a}=\left(x^{a}\right)^{*}$; since $\left\{\hat{e}^{1}, \ldots, \hat{e}^{n}\right\}$ is a basis of $M^{*}$ it follows that $\left\{\tilde{e}_{1}, \ldots, \tilde{e}_{n}\right\}$ is a basis of $M$. If we write $e_{i}=\tilde{e}_{a} x_{i}^{a}$, we have that

$$
h_{i j}=h\left(e_{i}, e_{j}\right)=h\left(e_{i}, \tilde{e}_{a} x_{j}^{a}\right)=h\left(e_{i}, \tilde{e}_{a}\right) x_{j}^{a}=x_{j}^{i},
$$

which $\left(\right.$ since $\left.\tilde{e}_{a}=\hat{h}^{-1}\left(\hat{e}^{a}\right)=e_{j} h^{j a}\right)$ implies that

$$
e_{i}=\tilde{e}_{a} x_{i}^{a}=e_{j}\left(h^{j a} h_{a i}\right),
$$

proving the statement.

The Christoffel symbols $\Gamma_{b c}^{a} \in \mathcal{A}$ for the Levi-Civita connection $\nabla$ are defined as the (unique) coefficients satisfying $\nabla_{b} e_{c}=e_{a} \Gamma_{b c}^{a}$. Using Koszul's formula, it is possible to derive an explicit formula for the Christoffel symbols $\Gamma_{b c}^{a}$ in terms of the components of the metric. By Koszul's formula we have

$$
h\left(e_{a} \Gamma_{b c}^{a}, e_{d}\right)=h\left(\nabla_{b} e_{c}, e_{d}\right)=\frac{1}{2}\left(\partial_{b} h_{c d}+\partial_{c} h_{b d}-\partial_{d} h_{b c}-h_{b r} f_{c d}^{r}+h_{c r} f_{d b}^{r}+h_{d r} f_{b c}^{r}\right),
$$


and since the right-hand side is hermitian it follows that

$$
h_{d a} \Gamma_{b c}^{a}=\frac{1}{2}\left(\partial_{b} h_{c d}+\partial_{c} h_{b d}-\partial_{d} h_{b c}-h_{b r} f_{c d}^{r}+h_{c r} f_{d b}^{r}+h_{d r} f_{b c}^{r}\right) .
$$

Multiplying from the left by the inverse metric coefficients $h^{p d}$ yields

$$
\Gamma_{b c}^{p}=\frac{1}{2} h^{p d}\left(\partial_{b} h_{c d}+\partial_{c} h_{b d}-\partial_{d} h_{b c}-h_{b r} f_{c d}^{r}+h_{c r} f_{d b}^{r}\right)+\mathbb{1} f_{b c}^{p}
$$

and, in particular, if $\left[\partial_{a}, \partial_{b}\right]=0$ for every $a, b \in\{1, \ldots, n\}$ then

$$
\Gamma_{b c}^{a}=\frac{1}{2} h^{a d}\left(\partial_{b} h_{c d}+\partial_{c} h_{b d}-\partial_{d} h_{b c}\right),
$$

corresponding to the classical formula from differential geometry.

\section{Projective real calculi}

Having discussed free real calculi it is possible to discuss real calculi over general projective modules in terms of their connection to free real calculi.

Definition 2.3.8. Let $C_{\mathcal{A}}=\left(\mathcal{A}, \mathfrak{g}_{D}, M, \varphi\right)$ be a real calculus and suppose that $M$ is a projective module. Then $C_{\mathcal{A}}$ is said to be a projective real calculus.

In general, given a free real calculus and a projection, it is easy to generate a projective real calculus as a "projection" of the free real calculus.

Proposition 2.3.9. Let $\tilde{C}_{\mathcal{A}}=\left(\mathcal{A}, \mathfrak{g}_{D}, \tilde{M}, \tilde{\varphi}\right)$ be a free real calculus and let $P$ : $\tilde{M} \rightarrow \tilde{M}$ be a projection. Then the structure $C_{\mathcal{A}}=\left(\mathcal{A}, \mathfrak{g}_{D}, P(\tilde{M}), P \circ \tilde{\varphi}\right)$ is a projective real calculus.

In light of the above proposition, it is natural to ask to which extent projective real calculi can be generated from free real calculi and projections in this way. Using the fact that any projective module is a projection of a free module, it is possible to use real calculus isomorphisms to show that every projective real calculus can be realized as the projection of a free real calculus.

Proposition 2.3.10 ([TN21] $)$. Let $C_{\mathcal{A}}=\left(\mathcal{A}, \mathfrak{g}_{D}, M, \varphi\right)$ be a real calculus, where $M$ is projective and $\operatorname{dim} \mathfrak{g}=n$. Then there exists a $\mathbb{R}$-linear map $\tilde{\varphi}: \mathfrak{g} \rightarrow \mathcal{A}^{n}$ and a projection $P: \mathcal{A}^{n} \rightarrow \mathcal{A}^{n}$ such that $\left(\mathcal{A}, \mathfrak{g}_{D}, \mathcal{A}^{n}, \tilde{\varphi}\right)$ is free and

$$
\left(\mathcal{A}, \mathfrak{g}_{D}, P\left(\mathcal{A}^{n}\right), P \circ \tilde{\varphi}\right) \simeq C_{\mathcal{A}}
$$

Before we discuss metric aspects of projective real calculi, we shall briefly describe how affine connections on these structures can be defined using the underlying structure of a free real calculus. The following proposition verifies that any connection on the projective module can indeed be defined through the use of a basis of the free module. 
Proposition 2.3.11. Let $P: \mathcal{A}^{n} \rightarrow \mathcal{A}^{n}$ be a projection. If $\tilde{\nabla}: \mathfrak{g} \times \mathcal{A}^{n} \rightarrow \mathcal{A}^{n}$ is an affine connection, then the map $\nabla: \mathfrak{g} \times P\left(\mathcal{A}^{n}\right) \rightarrow P\left(\mathcal{A}^{n}\right)$, defined by the formula

$$
\nabla_{\partial} m=P\left(\tilde{\nabla}_{\partial} m\right)
$$

is an affine connection. Conversely, for every affine connection $\nabla: \mathfrak{g} \times P\left(\mathcal{A}^{n}\right) \rightarrow$ $P\left(\mathcal{A}^{n}\right)$ there is an affine connection $\tilde{\nabla}: \mathfrak{g} \times \mathcal{A}^{n} \rightarrow \mathcal{A}^{n}$ such that

$$
\nabla_{\partial} m=P\left(\tilde{\nabla}_{\partial} m\right)
$$

for every $\partial \in \mathfrak{g}$ and $m \in P\left(\mathcal{A}^{n}\right)$.

Let $\tilde{C}_{\mathcal{A}}=\left(\mathcal{A}, \mathfrak{g}_{D}, \mathcal{A}^{n}, \tilde{\varphi}\right)$ be a free real calculus and let $\left(\tilde{C}_{\mathcal{A}}, \tilde{h}\right)$ be a free real metric calculus. If $P: \mathcal{A}^{n} \rightarrow \mathcal{A}^{n}$ is a projection it has already been established in Proposition 2.3.9 that we may generate the projective real calculus $C_{\mathcal{A}}=\left(\mathcal{A}, \mathfrak{g}_{D}, P\left(\mathcal{A}^{n}\right), P \circ \tilde{\varphi}\right)$ from $\tilde{C}_{\mathcal{A}}$. A natural next step is to ask under which conditions it is possible to generate a projective real metric calculus from $\left(\tilde{C}_{\mathcal{A}}, \tilde{h}\right)$, and if we assume that $P$ is orthogonal with respect to $\tilde{h}$ (i.e., $h\left(P\left(m_{1}\right), m_{2}\right)=h\left(m_{1}, P\left(m_{2}\right)\right)$ for every $\left.m_{1}, m_{2} \in \mathcal{A}^{n}\right)$ it is possible to make a few general statements.

Proposition 2.3.12. Let $C_{\mathcal{A}}=\left(\mathcal{A}, \mathfrak{g}_{D}, P\left(\mathcal{A}^{n}\right), P \circ \tilde{\varphi}\right)$ be a projective real calculus, where the projection $P$ is orthogonal with respect to the metric $\tilde{h}$ on $\mathcal{A}^{n}$. Then the following is true:

1. The map $h: P\left(\mathcal{A}^{n}\right) \times P\left(\mathcal{A}^{n}\right) \rightarrow \mathcal{A}$ given by $h\left(m_{1}, m_{2}\right)=\tilde{h}\left(m_{1}, m_{2}\right)$, $m_{1}, m_{2} \in P\left(\mathcal{A}^{n}\right)$, is a metric on $P\left(\mathcal{A}^{n}\right)$.

2. If $\tilde{\nabla}: \mathfrak{g} \times \mathcal{A}^{n} \rightarrow \mathcal{A}^{n}$ is an affine connection that is compatible with the metric $\tilde{h}$ then the affine connection $\nabla: \mathfrak{g} \times P\left(\mathcal{A}^{n}\right) \rightarrow P\left(\mathcal{A}^{n}\right)$, given by $\nabla_{\partial} m=P\left(\tilde{\nabla}_{\partial} m\right)$, is compatible with the metric $h$.

Left to answer is what conditions on $\tilde{h}$ and $P$ are needed to make sure that the projective real calculus $C_{\mathcal{A}}=\left(\mathcal{A}, \mathfrak{g}_{D}, P\left(\mathcal{A}^{n}\right), P \circ \tilde{\varphi}\right)$ together with the restriction $h$ of $\tilde{h}$ to $P\left(\mathcal{A}^{n}\right)$ constitute a real metric calculus. Although Proposition 2.3.12 ensures that $h$ is a metric on $P\left(\mathcal{A}^{n}\right)$, it is not guaranteed that $h$ is symmetric on $\varphi(\mathfrak{g})$, i.e., $h\left(\varphi\left(\partial_{1}\right), \varphi\left(\partial_{2}\right)\right)=h\left(\varphi\left(\partial_{2}\right), \varphi\left(\partial_{1}\right)\right)$ for every $\partial_{1}, \partial_{2} \in \mathfrak{g}$. To ensure this we need a few extra assumptions to hold for $\tilde{h}$ and $P$, and in the following statement we shall express both $\tilde{h}$ and $P$ with respect to a given basis $\left\{\hat{e}_{i}\right\}_{1}^{n}$ of $\mathcal{A}^{n}$. While the notation $\tilde{h}_{i j}=\tilde{h}\left(\hat{e}_{i}, \hat{e}_{j}\right)$ has been used earlier in the section on free real calculi, it is helpful to clarify how $P$ is expressed in terms of its coefficients $p_{j}^{i}$ with respect to the basis $\left\{\hat{e}_{i}\right\}_{1}^{n}$. Since $\mathcal{A}^{n}$ is a free module, we define $p_{j}^{i} \in \mathcal{A}, i, j=1, \ldots, n$, to be the unique coefficients such that

$$
P\left(\hat{e}_{j}\right)=\hat{e}_{i} p_{j}^{i}
$$

we note, in particular, that the coefficients $p_{j}^{i}$ satisfy the identity $p_{j}^{i} p_{k}^{j}=p_{k}^{i}$, since $P^{2}=P$ and $P$ is a module homomorphism. 
Proposition 2.3.13. Let $\tilde{h}$ be a metric on $\mathcal{A}^{n}$ that is symmetric for a given basis $\left\{\hat{e}_{k}\right\}_{1}^{n}$ and let $P$ be an orthogonal projection with respect to $\tilde{h}$. If $C_{\mathcal{A}}=$ $\left(\mathcal{A}, \mathfrak{g}_{D}, P\left(\mathcal{A}^{n}\right), \varphi\right)$ is a projective real calculus such that $e_{k}:=\varphi\left(\partial_{k}\right)=P\left(\hat{e}_{k}\right)$ and $h$ is the restriction of $\tilde{h}$ to $P\left(\mathcal{A}^{n}\right)$, then $\left(C_{\mathcal{A}}, h\right)$ is a real metric calculus if and only if for any pair indices $i$ and $j$,

$$
\tilde{h}_{j k} p_{i}^{k}=\tilde{h}_{i k} p_{j}^{k}
$$





\section{3 - A theory of noncommutative embeddings}

Real calculus homomorphisms can be viewed from both a geometric and an algebraic perspective, and as outlined in ATN21 real calculus homomorphisms are intimately connected to the concept of embeddings when viewed from a geometric perspective. As was briefly touched upon in Chapter 2, Definition 2.2.1 of real calculus homomorphisms is originally inspired from this perspective. Thus, we shall outline an embedding theory based on real calculus homomorphisms in this chapter, with a special emphasis of isometric embeddings of pseudo-Riemannian real calculi.

\subsection{General embeddings}

In order to understand how the notion of embeddings can be generalized to the context of real calculi, it is helpful to consider how embeddings work for classical manifolds. Let $\phi_{0}: \Sigma^{\prime} \hookrightarrow \Sigma$ be an embedding of $\Sigma^{\prime}$ into $\Sigma$ and let $\phi: C^{\infty}(\Sigma) \rightarrow$ $C^{\infty}\left(\Sigma^{\prime}\right)$ be the corresponding homomorphism of the algebras of smooth functions.

First of all, there is no natural map from $\operatorname{Vect}(\Sigma)$ to $\operatorname{Vect}\left(\Sigma^{\prime}\right)$ since a vector field $X \in \operatorname{Vect}(\Sigma)$ at a point $p \in \phi_{0}\left(\Sigma^{\prime}\right)$ might not lie in $T_{p} \Sigma^{\prime}$ (regarded as a subspace of $\left.T_{p} \Sigma\right)$. However, vector fields which are tangent to $\Sigma^{\prime}$ in this sense may be restricted to $\Sigma^{\prime}$. On the other hand, any vector field $X^{\prime} \in \operatorname{Vect}\left(\Sigma^{\prime}\right)$ (assuming $\Sigma^{\prime}$ to be closed) can be extended to a smooth vector field $X \in \operatorname{Vect}(\Sigma)$ such that $\left.X\right|_{\Sigma^{\prime}}=X^{\prime}$. In light of the isomorphism between vector fields and derivations, it is natural to have a map $\psi: \operatorname{Der}\left(C^{\infty}\left(\Sigma^{\prime}\right)\right) \rightarrow \operatorname{Der}\left(C^{\infty}(\Sigma)\right)$ correspond to a choice of extension of vector fields on $\Sigma^{\prime}$. The map $\hat{\psi}$, corresponding to the restriction of vector fields on $\Sigma$ which are tangent to $\Sigma^{\prime}$, can then be seen as "a left inverse" of $\psi$.

In the language of real calculi we have

$$
\begin{array}{ccc}
\mathcal{A}=C^{\infty}(\Sigma) & \stackrel{\phi}{\longrightarrow} \quad \mathcal{A}^{\prime}=C^{\infty}\left(\Sigma^{\prime}\right) \\
\mathfrak{g}=\operatorname{Der}(\mathcal{A}) \quad \stackrel{\psi}{\longleftarrow} & \mathfrak{g}^{\prime}=\operatorname{Der}\left(\mathcal{A}^{\prime}\right) \\
M=\operatorname{Vect}(\Sigma) \supseteq M_{\Psi} & \stackrel{\hat{\psi}}{\longrightarrow} & M^{\prime}=\operatorname{Vect}\left(\Sigma^{\prime}\right),
\end{array}
$$

i.e., an embedding $\phi_{0}: \Sigma^{\prime} \hookrightarrow \Sigma$ gives rise to a corresponding real calculus homomorphism $(\phi, \psi, \hat{\psi})$.

Using the above analogy between real calculus homomorphisms and embeddings we shall now make the distinction between a general homomorphism and an embedding precise. In the analogy above, a necessary condition for $\phi_{0}: \Sigma^{\prime} \hookrightarrow \Sigma$ to 
be an embedding is that $\phi_{0}$ is injective; this corresponds to $\phi: C^{\infty}(\Sigma) \rightarrow C^{\infty}\left(\Sigma^{\prime}\right)$ being surjective.

Definition 3.1.1. Let $(\phi, \psi, \hat{\psi}): C_{\mathcal{A}} \rightarrow C_{\mathcal{A}^{\prime}}$ be a real calculus homomorphism. If $\phi$ is surjective and there exists a submodule $\tilde{M} \subseteq M$ such that $M=M_{\Psi} \oplus \tilde{M}$, then $(\phi, \psi, \hat{\psi})$ is called an embedding. Furthermore, if there exist metrics $h$ and $h^{\prime}$ such that $(\phi, \psi, \hat{\psi}):\left(C_{\mathcal{A}}, h\right) \rightarrow\left(C_{\mathcal{A}^{\prime}}, h^{\prime}\right)$ is a real metric calculus homomorphism and $M=M_{\Psi} \oplus M_{\Psi}^{\perp}$, then the embedding is said to be isometric.

The following statement gives further motivation for Definition 3.1.1.

Proposition 3.1.2 ([ATN21]). Assume that $(\phi, \psi, \hat{\psi}): C_{\mathcal{A}} \rightarrow C_{\mathcal{A}^{\prime}}$ is a real calculus homomorphism, where $\phi$ is surjective. Then $\psi$ is injective and $\hat{\psi}$ is surjective.

Thus, if $(\phi, \psi, \hat{\psi}): C_{\mathcal{A}} \rightarrow C_{\mathcal{A}^{\prime}}$ is an embedding, then $\psi$ is injective in analogy with the injectivity of the tangent map of an embedding. Proposition 3.1.2 also implies that every element $m^{\prime} \in M^{\prime}$ has at least one extension in $M$, corresponding to the geometric situation where vector fields of the embedded manifold can be extended to vector fields in the ambient space.

Definition 3.1.3. Let $(\phi, \psi, \hat{\psi}): C_{\mathcal{A}} \rightarrow C_{\mathcal{A}^{\prime}}$ be an embedding. Then, if $m \in M_{\Psi}$ is such that $\hat{\psi}(m)=m^{\prime}$ then $m$ is called an extension of $m^{\prime}$. The set of extensions of $m^{\prime}$ is denoted by $\operatorname{Ext}_{\Psi}\left(m^{\prime}\right)$.

\subsection{Isometric embeddings}

Given an embedding $(\phi, \psi, \hat{\psi}): C_{\mathcal{A}} \rightarrow C_{\mathcal{A}^{\prime}}$, we shall define the $\mathcal{A}$-linear projection $P: M \rightarrow M_{\Psi}$ as

$$
P\left(m_{\Psi} \oplus \tilde{m}\right)=m_{\Psi}
$$

with respect to the decomposition $M=M_{\Psi} \oplus \tilde{M}$. The complementary projection will be denoted by $\Pi=\mathbb{1}-P$.

In analogy with classical Riemannian submanifold theory, one decomposes the Levi-Civita connection in its tangential and normal parts. Let $\left(C_{\mathcal{A}}, h, \nabla\right)$ and $\left(C_{\mathcal{A}^{\prime}}, h^{\prime}, \nabla^{\prime}\right)$ be psuedo-Riemannian calculi and assume that $(\phi, \psi, \hat{\psi}):\left(C_{\mathcal{A}}, h\right) \rightarrow$ $\left(C_{\mathcal{A}^{\prime}}, h^{\prime}\right)$ is an isometric embedding and write

$$
\begin{aligned}
& \nabla_{\psi(\delta)} m=L(\delta, m)+\alpha(\delta, m) \\
& \nabla_{\psi(\delta)} \xi=-A_{\xi}(\delta)+D_{\delta} \xi
\end{aligned}
$$

for $\delta \in \mathfrak{g}^{\prime}, m \in M_{\Psi}$ and $\xi \in M_{\Psi}^{\perp}$, with

$$
\begin{array}{ll}
L(\delta, m)=P\left(\nabla_{\psi(\delta)} m\right) & \alpha(\delta, m)=\Pi\left(\nabla_{\psi(\delta)} m\right) \\
A_{\xi}(\delta)=-P\left(\nabla_{\psi(\delta)} \xi\right) & D_{\delta} \xi=\Pi\left(\nabla_{\psi(\delta)} \xi\right) .
\end{array}
$$

In classical geometry, (3.1) is called Gauss'formula and 3.2 is called Weingarten's formula. Furthermore, $\alpha: \mathfrak{g}^{\prime} \times M_{\Psi} \rightarrow M_{\Psi}^{\perp}$ is called the second fundamental form 
and $A: \mathfrak{g}^{\prime} \times M_{\Psi}^{\perp} \rightarrow M_{\Psi}$ is called the Weingarten map. The maps $L, \alpha, A_{\xi}$ and $D_{\delta}$ have similar properties to their classical counterparts, as the following statements show.

Proposition 3.2.1. If $\delta \in \mathfrak{g}^{\prime}$ and $m \in \operatorname{Ext}_{\Psi}\left(m^{\prime}\right)$ then $L(\delta, m) \in \operatorname{Ext}_{\Psi}\left(\nabla_{\delta}^{\prime} m^{\prime}\right)$.

Note that the above proposition requires that both $\left(C_{\mathcal{A}}, h, \nabla\right)$ and $\left(C_{\mathcal{A}^{\prime}}, h^{\prime}, \nabla^{\prime}\right)$ are pseudo-Riemannian. In general, if $\left(C_{\mathcal{A}}, h, \nabla\right)$ is pseudo-Riemannian and $\left(C_{\mathcal{A}^{\prime}}, h^{\prime}\right)$ is isometrically embedded into $\left(C_{\mathcal{A}}, h\right)$ then the above result cannot be used to conclude that there exists a connection $\nabla^{\prime}$ such that $\left(C_{\mathcal{A}^{\prime}}, h^{\prime}, \nabla^{\prime}\right)$ is pseudoRiemannian. Indeed, since general modules are not required to have a basis it is not clear that there exists a well-defined connection $\nabla^{\prime}: \mathfrak{g}^{\prime} \times M^{\prime}$ such that $\nabla_{\delta}^{\prime} m^{\prime}=\hat{\psi}(L(\delta, m))$ for every $m \in \operatorname{Ext}_{\Psi}\left(m^{\prime}\right)$. However, if such a connection $\nabla^{\prime}$ exists then one checks that $\left(C_{\mathcal{A}^{\prime}}, h^{\prime}, \nabla^{\prime}\right)$ is pseudo-Riemannian by using Koszul's formula in combination with the fact that $h^{\prime}\left(\hat{\psi}\left(m_{1}\right), \hat{\psi}\left(m_{2}\right)\right)=\phi\left(h\left(m_{1}, m_{2}\right)\right)$ for all $m_{1}, m_{2} \in M_{\Psi}$.

Proposition 3.2.2. If $\delta_{1}, \delta_{2} \in \mathfrak{g}^{\prime}, a^{1}, a^{2} \in A$ and $\lambda_{1}, \lambda_{2} \in \mathbb{R}$ then

$$
\begin{aligned}
& \alpha\left(\delta_{1}, \Psi\left(\delta_{2}\right)\right)=\alpha\left(\delta_{2}, \Psi\left(\delta_{1}\right)\right) \\
& \alpha\left(\lambda_{1} \delta_{1}+\lambda_{2} \delta_{2}, m_{1}\right)=\lambda_{1} \alpha\left(\delta_{1}, m_{1}\right)+\lambda_{2} \alpha\left(\delta_{2}, m_{1}\right) \\
& \alpha\left(\delta_{1}, m_{1} a^{1}+m_{2} a^{2}\right)=\alpha\left(\delta_{1}, m_{1}\right) a^{1}+\alpha\left(\delta_{1}, m_{2}\right) a^{2}
\end{aligned}
$$

for $m_{1}, m_{2} \in M_{\Psi}$.

Proposition 3.2.3. If $\delta \in \mathfrak{g}^{\prime}, m \in M_{\Psi}$ and $\xi \in M_{\Psi}^{\perp}$ then

$$
h\left(A_{\xi}(\delta), m\right)=h(\xi, \alpha(\delta, m)) .
$$

Thus, the Weingarten map $A_{\xi}$ and the second fundamental form $\alpha$ are connected in the expected way. Finally, we note that the map $D_{\delta}$ has the properties of an affine connection; in differential geometry, $D_{\delta}$ is usually identified with a connection on the normal bundle of the submanifold.

Proposition 3.2.4. If $\delta_{1}, \delta_{2} \in \mathfrak{g}^{\prime}, \xi_{1}, \xi_{2} \in M_{\Psi}^{\perp}, \lambda \in \mathbb{R}$ and $a \in \mathcal{A}$ then

1. $D_{\delta_{1}}\left(\xi_{1}+\xi_{2}\right)=D_{\delta_{1}} \xi_{1}+D_{\delta_{1}} \xi_{2}$,

2. $D_{\lambda \delta_{1}+\delta_{2}} \xi_{1}=\lambda D_{\delta_{1}} \xi_{1}+D_{\delta_{2}} \xi_{1}$,

3. $D_{\delta_{1}}\left(\xi_{1} a\right)=\left(D_{\delta_{1}} \xi_{1}\right) a+\xi_{1} \psi\left(\delta_{1}\right)(a)$.

Having discussed aspects of the Levi-Civita connection with regards to isometric embeddings we wish to discuss minimality of an embedding, which is illustrated in the next chapter where the noncommutative torus is minimally embedded into the noncommutative 3 -sphere. Let $\left(C_{\mathcal{A}}, h\right)$ and $\left(C_{\mathcal{A}^{\prime}}, h^{\prime}\right)$ be free real metric calculi and let $(\phi, \psi, \hat{\psi}):\left(C_{\mathcal{A}}, h\right) \rightarrow\left(C_{\mathcal{A}^{\prime}}, h^{\prime}\right)$ be an isometric embedding. Since $\psi$ is injective, it is straightforward to check that if $\left\{\delta_{1}, \ldots, \delta_{n}\right\}$ is a basis of $\mathfrak{g}^{\prime}$, then 
$\left\{\Psi\left(\delta_{1}\right), \ldots, \Psi\left(\delta_{n}\right)\right\}$ is a basis of $M_{\Psi}$, implying that $M_{\Psi}$ is a free module of rank $n$. We are now ready to define mean curvature, as well as minimality, of an embedding of free real metric calculi. Since we are working with extensions of vector fields on the embedded manifold $\Sigma^{\prime}$, rather than tangent vectors at points on $\Sigma^{\prime}$, it is more natural to consider the restriction (to $\Sigma^{\prime}$ ) of the inner product of the mean curvature vector with an arbitrary vector, rather than the mean curvature vector itself.

Definition 3.2.5. Let $\left(C_{\mathcal{A}}, h\right)$ and $\left(C_{\mathcal{A}^{\prime}}, h^{\prime}\right)$ be free real metric calculi, and let $(\phi, \psi, \hat{\psi}):\left(C_{\mathcal{A}}, h\right) \rightarrow\left(C_{\mathcal{A}^{\prime}}, h^{\prime}\right)$ be an isometric embedding. Given a basis $\left\{\delta_{1}, \ldots, \delta_{n}\right\}$ for $\mathfrak{g}^{\prime}$, the mean curvature $H_{\mathcal{A}^{\prime}}$ of the embedding with respect to $\left\{\delta_{1}, \ldots, \delta_{n}\right\}$ is defined as

$$
H_{\mathcal{A}^{\prime}}(m)=\phi\left(h\left(m, \alpha\left(\delta_{i}, \Psi\left(\delta_{j}\right)\right)\right)\right)\left(h^{\prime}\right)^{i j}
$$

Giving trivially $H_{\mathcal{A}^{\prime}}(m)=0$ for every $m \in M_{\Psi}$. An embedding is said to be minimal if $H_{\mathcal{A}^{\prime}}(\xi)=0$ for every $\xi \in M_{\Psi}^{\perp}$ as well, i.e. if $H_{\mathcal{A}^{\prime}} \equiv 0$.

Although the above definition of mean curvature is given with respect to a basis, the mean curvature of the embedding is independent of the specific choice of basis. We state this as follows.

Proposition 3.2.6. Let $\left(C_{\mathcal{A}}, h\right)$ and $\left(C_{\mathcal{A}^{\prime}}, h^{\prime}\right)$ be free real metric calculi, and let $(\phi, \psi, \hat{\psi}):\left(C_{\mathcal{A}}, h\right) \rightarrow\left(C_{\mathcal{A}^{\prime}}, h^{\prime}\right)$ be an isometric embedding. Moreover, let $\left\{\delta_{i}\right\}_{1}^{n}$ and $\left\{\tilde{\delta}_{i}\right\}_{1}^{n}$ be bases of $\mathfrak{g}^{\prime}$, and let $h_{i j}^{\prime}$ and $\tilde{h}_{i j}^{\prime}$ denote the respective components of $h^{\prime}$ with respect to these bases. Then

$$
\phi\left(h\left(m, \alpha\left(\tilde{\delta}_{i}, \Psi\left(\tilde{\delta}_{j}\right)\right)\right)\right)\left(\tilde{h}^{\prime}\right)^{i j}=\phi\left(h\left(m, \alpha\left(\delta_{i}, \Psi\left(\delta_{j}\right)\right)\right)\right)\left(h^{\prime}\right)^{i j},
$$

i.e., the mean curvature $H_{\mathcal{A}^{\prime}}$ is independent of the specific choice of basis.

Proof. Since $\left\{\delta_{i}\right\}_{1}^{n}$ and $\left\{\tilde{\delta}_{i}\right\}_{1}^{n}$ be bases of $\mathfrak{g}^{\prime}$ there exists a real invertible matrix $A$ such that $\tilde{h}^{\prime}=A h^{\prime} A^{T}$ or, using index notation, $\tilde{h}_{i j}^{\prime}=A_{i}^{k} h_{k l}^{\prime} A_{j}^{l}$. This implies that the components of the inverse metric are related as follows

$$
\left(\tilde{h}^{\prime}\right)^{i j}=\left(A^{-1}\right)_{k}^{i}\left(h^{\prime}\right)^{k l}\left(A^{-1}\right)_{l}^{j},
$$

implying that

$$
\begin{aligned}
\phi\left(h\left(m, \alpha\left(\tilde{\delta}_{i}, \Psi\left(\tilde{\delta}_{j}\right)\right)\right)\right)\left(\tilde{h}^{\prime}\right)^{i j} & =\phi\left(h\left(m, \alpha\left(A_{i}^{k} \delta_{k}, \Psi\left(A_{j}^{l} \delta_{l}\right)\right)\right)\right)\left(A^{-1}\right)_{p}^{i}\left(h^{\prime}\right)^{p q}\left(A^{-1}\right)_{q}^{j} \\
& =A_{i}^{k}\left(A^{-1}\right)_{p}^{i} A_{j}^{l}\left(A^{-1}\right)_{q}^{j} \phi\left(h\left(m, \alpha\left(\delta_{k}, \Psi\left(\delta_{l}\right)\right)\right)\right)\left(h^{\prime}\right)^{k l} \\
& =\phi\left(h\left(m, \alpha\left(\delta_{k}, \Psi\left(\delta_{l}\right)\right)\right)\left(h^{\prime}\right)^{k l},\right.
\end{aligned}
$$

as desired.

As a final note on this topic, we may technically also view projective real calculi as embedded into free real calculi. However, this perspective does not give us any additional insights as we are unable to use the theory of isometric embeddings when discussing projective real calculi as embedded into free real calculi. This is because 
of the simple fact that if $\left(\operatorname{id}_{\mathcal{A}}, \mathrm{id}_{\mathfrak{g}}, P\right)$ is a real metric calculus homomorphism from a free real calculus to a projective real calculus, then it is by necessity also an isomorphism, which means the projective real metric calculus being studied is, in fact, free itself.

Proposition 3.2.7. Let $C_{\mathcal{A}}=\left(\mathcal{A}, \mathfrak{g}_{D}, P\left(\mathcal{A}^{n}\right), P \circ \tilde{\varphi}\right)$ be a projective real calculus. Then $C_{\mathcal{A}}$ is embedded into the free real calculus $\tilde{C}_{\mathcal{A}}=\left(\mathcal{A}, \mathfrak{g}_{D}, \mathcal{A}^{n}, \tilde{\varphi}\right)$ by the real calculus homomorphism $\left(\operatorname{id}_{\mathcal{A}}, \operatorname{id}_{\mathfrak{g}}, P\right): \tilde{C}_{\mathcal{A}} \rightarrow C_{\mathcal{A}}$, and if there exist metrics $\tilde{h}$ and $h$ such that $\left(\operatorname{id}_{\mathcal{A}}, \operatorname{id}_{\mathfrak{g}}, P\right):\left(\tilde{C}_{\mathcal{A}}, \tilde{h}\right) \rightarrow\left(C_{\mathcal{A}}, h\right)$ is an isometric embedding, then $\left(\operatorname{id}_{\mathcal{A}}, \operatorname{id}_{\mathfrak{g}}, P\right)$ is an isomorphism of real calculi.

Proof. That $\mathrm{id}_{\mathcal{A}}$ and $\mathrm{id}_{\mathfrak{g}}$ are compatible is trivial to check. Moreover, since $P$ is a projection it is a module homomorphism; together with the fact that $\varphi(\partial)=$ $P(\tilde{\varphi}(\partial))=P(\Psi(\partial))$ for all $\partial \in \mathfrak{g}$, this implies that $P$ is compatible with $\operatorname{id}_{\mathcal{A}}$ and $\operatorname{id}_{\mathfrak{g}}$. Since $\operatorname{id}_{\mathcal{A}}$ is surjective and $\mathcal{A}^{n}=\mathcal{A}_{\Psi}^{n}=\mathcal{A}_{\Psi}^{n} \oplus 0$, it follows that $\left(\operatorname{id}_{\mathcal{A}}, \operatorname{id}_{\mathfrak{g}}, P\right)$ is an embedding.

For the second statement, all that is left to prove is that $P$ is injective since it is obviously surjective. Let $\tilde{m}$ belong to the kernel of $P$. Then, since $\left(\operatorname{id}_{\mathcal{A}}, \operatorname{id}_{\mathfrak{g}}, P\right)$ is an isometric embedding, it follows that

$$
0=h(0, P(\tilde{n}))=h(P(\tilde{m}), P(\tilde{n}))=\tilde{h}(\tilde{m}, \tilde{n})
$$

for every $\tilde{n} \in \mathcal{A}_{\Psi}^{n}=\mathcal{A}^{n}$. Since $\tilde{h}$ is a metric, this implies that $\tilde{m}=0$ and the statement follows. 



\section{4 - The noncommutative torus and 3-sphere}

We shall illustrate the theory of isometric embeddings developed in Chapter 3 by embedding the noncommutative torus $T_{\theta}^{2}$ into the noncommutative 3 -sphere $S_{\theta}^{3}$. These noncommutative manifolds are well known and understood, and since they are closely related to their classical counterparts $T^{2}$ and $S^{3}$ it is natural to suspect that $T_{\theta}^{2}$ can be minimally embedded into $S_{\theta}^{3}$. While both $T_{\theta}^{2}$ and $S_{\theta}^{3}$ are well known in the context of noncommutative geometry in general, the framework of real calculi cannot be considered standard enough for it to be widely known how these noncommutative manifolds can be realized as real calculi in a natural way. Therefore, in the below descriptions of $T_{\theta}^{2}$ and $S_{\theta}^{3}$ we shall begin by giving a brief outline of their classical counterparts, which helps to clarify how the real calculi over $T_{\theta}^{2}$ and $S_{\theta}^{3}$ constructed below can be viewed as natural noncommutative analogues of $T^{2}$ and $S^{3}$ within the framework. For a more detailed description of real calculi over $T_{\theta}^{2}$ and $S_{\theta}^{3}$, see AW17b.

\subsection{The noncommutative torus}

In a classical geometric setting, the Clifford torus $T^{2}$ as embedded in $\mathbb{R}^{4}$ can be parameterized by

$$
\vec{x}=\left(x^{1}, x^{2}, x^{3}, x^{4}\right)=\left(\cos \varphi_{1}, \sin \varphi_{1}, \cos \varphi_{2}, \sin \varphi_{2}\right),
$$

where $\varphi_{1}$ and $\varphi_{2}$ are real coordinates between 0 and $2 \pi$ (working in complex coordinates, we may set $\left.u=x^{1}+i x^{2}, v=x^{3}+i x^{4}\right)$. With $\partial_{1}=\partial_{\varphi_{1}}$ and $\partial_{2}=\partial_{\varphi_{2}}$ this means that the tangent space at a point is spanned by

$$
\begin{aligned}
& \partial_{1} \vec{x}=\left(-\sin \varphi_{1}, \cos \varphi_{1}, 0,0\right)=\left(-x^{2}, x^{1}, 0,0\right) \\
& \partial_{2} \vec{x}=\left(0,0,-\sin \varphi_{2}, \cos \varphi_{2}\right)=\left(0,0,-x^{4}, x^{3}\right) .
\end{aligned}
$$

Working in complex coordinates, we may set $u=x^{1}+i x^{2}, v=x^{3}+i x^{4}$ and this yields

$$
\begin{array}{llrl}
\partial_{1} u & =i u & \partial_{1} v & =0 \\
\partial_{2} u & =0 & \partial_{2} v & =i v .
\end{array}
$$

As an analogy to the Clifford torus in the noncommutative setting, we consider the noncommutative torus $T_{\theta}^{2}$ as the unital *-algebra generated by two unitary operators $U, V$ satisfying the relation $V U=q U V$, where $q=e^{2 \pi i \theta}$. Introducing 
the notation

$$
\begin{aligned}
X^{1} & =\frac{1}{2}\left(U+U^{*}\right) & X^{2} & =\frac{1}{2 i}\left(U-U^{*}\right) \\
X^{3} & =\frac{1}{2}\left(V+V^{*}\right) & X^{4} & =\frac{1}{2 i}\left(V-V^{*}\right)
\end{aligned}
$$

yields $\mathbb{1}=U U^{*}=\left(X^{1}\right)^{2}+\left(X^{2}\right)^{2}$ and $\mathbb{1}=V V^{*}=\left(X^{3}\right)^{2}+\left(X^{4}\right)^{2}$, and we note in particular that $\left(X^{j}\right)^{*}=X^{j}$ for $j=1,2,3,4$.

In analogy with the geometrical setting, let $M^{\prime}$ be the (right) submodule of $\left(T_{\theta}^{2}\right)^{4}$ generated by

$$
\begin{aligned}
& e_{1}=\left(-X^{2}, X^{1}, 0,0\right) \\
& e_{2}=\left(0,0,-X^{4}, X^{3}\right) .
\end{aligned}
$$

Worth noting is that $M^{\prime}$ is a free $T_{\theta}^{2}$-module, since $e_{1}$ and $e_{2}$ form a basis for $M^{\prime}$ :

$$
\begin{aligned}
e_{1} a+e_{2} b=0 \Longleftrightarrow\left(-X^{2} a, X^{1} a,\right. & \left.-X^{4} b, X^{3} b\right)=(0,0,0,0) \\
& \Longleftrightarrow\left\{\begin{array}{l}
\left(\left(X^{1}\right)^{2}+\left(X^{2}\right)^{2}\right) a=U U^{*} a=a=0 \\
\left.\left(X^{3}\right)^{2}+\left(X^{4}\right)^{2}\right) b=V V^{*} b=b=0 .
\end{array}\right.
\end{aligned}
$$

Next, we let $\mathfrak{g}^{\prime}$ be the (real) Lie algebra generated by the two hermitian derivations $\delta_{1}, \delta_{2}$, given by

$$
\begin{array}{ll}
\delta_{1} U=i U & \delta_{1} V=0 \\
\delta_{2} U=0 & \delta_{2} V=i V,
\end{array}
$$

with Lie bracket $\left[\delta_{1}, \delta_{2}\right]=0$. Lastly, we let $\varphi^{\prime}: \mathfrak{g}^{\prime} \rightarrow M^{\prime}$ be defined as $\varphi^{\prime}\left(\delta_{j}\right)=e_{j}$ for $j=1,2$ and extended by $\mathbb{R}$-linearity, which in turn means that $M^{\prime}$ is generated by $\varphi^{\prime}\left(\mathfrak{g}^{\prime}\right)$ as a $T_{\theta}^{2}$-module. The resulting real calculus $C_{T_{\theta}^{2}}=\left(T_{\theta}^{2}, \mathfrak{g}^{\prime}, M^{\prime}, \varphi^{\prime}\right)$ is a free real calculus which can be seen as a noncommutative analogue to the differential structure of the Clifford torus $T^{2}$.

\subsection{The noncommutative 3-sphere}

The 3-sphere $S^{3}$ can be described as embedded in $\mathbb{C}^{2}$ by the two complex coordinates $z=x^{1}+i x^{2}$ and $w=x^{3}+i x^{4}$ satisfying $|z|^{2}+|w|^{2}=1 ; z$ and $w$ are represented by the coordinates $\xi_{1}, \xi_{2}, \eta$ :

$$
\begin{gathered}
z=e^{i \xi_{1}} \sin \eta \\
w=e^{i \xi_{2}} \cos \eta
\end{gathered}
$$

i.e.

$$
\begin{array}{ll}
x^{1}=\cos \xi_{1} \sin \eta & x^{2}=\sin \xi_{1} \sin \eta \\
x^{3}=\cos \xi_{2} \cos \eta & x^{4}=\sin \xi_{2} \cos \eta
\end{array}
$$


With $\partial_{1}=\partial_{\xi_{1}}$ and $\partial_{2}=\partial_{\xi_{2}}$ we get that at every point where $0<\xi_{1}, \xi_{2}<2 \pi$ and $0<\eta<\pi / 2$, the tangent space is spanned by the vectors

$$
\begin{aligned}
& E_{1}=\partial_{1}\left(x^{1}, x^{2}, x^{3}, x^{4}\right)=\left(-x^{2}, x^{1}, 0,0\right) \\
& E_{2}=\partial_{2}\left(x^{1}, x^{2}, x^{3}, x^{4}\right)=\left(0,0,-x^{4}, x^{3}\right) \\
& E_{\eta}=\partial_{\eta}\left(x^{1}, x^{2}, x^{3}, x^{4}\right)=\left(\cos \xi_{1} \cos \eta, \sin \xi_{1} \cos \eta,-\cos \xi_{2} \sin \eta,-\sin \xi_{2} \sin \eta\right),
\end{aligned}
$$

however, $E_{\eta}$ is not very practical to use in calculations. Therefore, instead of $\partial_{\eta}$, one may use the derivation $\partial_{3}=|z||w| \partial_{\eta}$ corresponding to the vector

$$
E_{3}=\partial_{3}\left(x^{1}, x^{2}, x^{3}, x^{4}\right)=\left(x^{1}|w|^{2}, x^{2}|w|^{2},-x^{3}|z|^{2},-x^{4}|z|^{2}\right),
$$

which can be used together with $E_{1}$ and $E_{2}$ to span the tangent space.

Returning to $z$ and $w$, one gets that

$$
\begin{array}{ll}
\partial_{1}(z)=i z & \partial_{1}(w)=0 \\
\partial_{2}(z)=0 & \partial_{2}(w)=i w \\
\partial_{3}(z)=z|w|^{2} & \partial_{2}(w)=-w|z|^{2},
\end{array}
$$

and with respect to the basis $\left\{E_{1}, E_{2}, E_{3}\right\}$ of the tangent space of $S^{3}$ at a given point (where $0<\xi_{1}, \xi_{2}<2 \pi$ and $0<\eta<\pi / 2$ ) the induced metric becomes

$$
\left(h_{a b}\right)=\left(h\left(E_{a}, E_{b}\right)\right)=\left(\begin{array}{ccc}
|z|^{2} & 0 & 0 \\
0 & |w|^{2} & 0 \\
0 & 0 & |z|^{2}|w|^{2}
\end{array}\right) .
$$

As a noncommutative analogue to the above characterization of the classical 3 -sphere, we consider the noncommutative 3 -sphere $S_{\theta}^{3}$ to be the unital *-algebra generated by $Z, Z^{*}, W, W^{*}$ satisfying the relations

$$
\begin{aligned}
& W Z=q Z W \quad W^{*} Z=\bar{q} Z W^{*} \quad W Z^{*}=\bar{q} Z^{*} W \quad W^{*} Z^{*}=q Z^{*} W^{*} \\
& Z^{*} Z=Z Z^{*} \quad W^{*} W=W W^{*} \quad W W^{*}=\mathbb{1}-Z Z^{*},
\end{aligned}
$$

where $q=e^{2 \pi i \theta}$.

Similar to what was done for the torus we introduce the notation

$$
\begin{aligned}
X^{1} & =\frac{1}{2}\left(Z+Z^{*}\right) & X^{2} & =\frac{1}{2 i}\left(Z-Z^{*}\right) \\
X^{3} & =\frac{1}{2}\left(W+W^{*}\right) & X^{4} & =\frac{1}{2 i}\left(W-W^{*}\right) \\
|Z|^{2} & =Z Z^{*} & |W|^{2} & =W W^{*},
\end{aligned}
$$

and we get that $|Z|^{2}=\left(X^{1}\right)^{2}+\left(X^{2}\right)^{2}$ and $|W|^{2}=\left(X^{3}\right)^{2}+\left(X^{4}\right)^{2}$; the elements $|Z|^{2}$ and $|W|^{2}$ both lie in the center of $S_{\theta}^{3}$, and neither of them is a zero divisor (see AW17b]). As for $T_{\theta}^{2}$ we want to construct a real metric calculus for $S_{\theta}^{3}$, and we want it to be closely related to the differential structure of the 3-sphere presented above. Thus, let $M$ be the right module over $S_{\theta}^{3}$ generated by $\left\{E_{1}, E_{2}, E_{3}\right\}$ where

$$
\begin{aligned}
& E_{1}=\left(-X^{2}, X^{1}, 0,0\right) \\
& E_{2}=\left(0,0,-X^{4}, X^{3}\right) \\
& E_{3}=\left(X^{1}|W|^{2}, X^{2}|W|^{2},-X^{3}|Z|^{2},-X^{4}|Z|^{2}\right) .
\end{aligned}
$$


Proposition 4.2.1. ([AW17b] $) M=\left\{E_{1} a+E_{2} b+E_{3} c: a, b, c \in S_{\theta}^{3}\right\}$ is a free right $S_{\theta}^{3}$-module with a basis given by the elements $\left\{E_{1}, E_{2}, E_{3}\right\}$.

Proposition 4.2.2. ( $\mathrm{AW} 17 \mathrm{~b}]$ ) There exist hermitian derivations $\partial_{1}, \partial_{2}, \partial_{3} \in$ $\operatorname{Der}\left(S_{\theta}^{3}\right)$ such that such that

$$
\begin{aligned}
& \partial_{1}(Z)=i Z \\
& \partial_{1}(W)=0 \\
& \partial_{2}(Z)=0 \\
& \partial_{2}(W)=i W \\
& \partial_{3}(Z)=Z|W|^{2} \\
& \partial_{3}(W)=-W|Z|^{2},
\end{aligned}
$$

and $\left[\partial_{a}, \partial_{b}\right]=0$ for $a, b=1,2,3$.

Let $\mathfrak{g}$ be the (real) Lie algebra generated by $\partial_{1}, \partial_{2}$ and $\partial_{3}$ in the above proposition, and define $\varphi: \mathfrak{g} \rightarrow M$ as the linear map over $\mathbb{R}$ given by $\varphi\left(\partial_{a}\right)=E_{a}$ for $a=1,2,3$. The real calculus $C_{S_{\theta}^{3}}=\left(S_{\theta}^{3}, \mathfrak{g}, M, \varphi\right)$ over $S_{\theta}^{3}$ is then a free real calculus which can be seen as a noncommutative analogue to the differential structure of $S^{3}$.

In order to isometrically embed the noncommutative torus into the noncommutative 3 -sphere, a metric on $M$ will be needed, and the choice for this thesis will be the hermitian form $h$ given by

$$
h(m, n)=\sum_{a, b=1}^{3}\left(m^{a}\right)^{*} h_{a, b} n^{b},
$$

where $m=E_{a} m^{a}, n=E_{b} n^{b}$ and

$$
\left(h_{a, b}\right)=H\left(\begin{array}{ccc}
|Z|^{2} & 0 & 0 \\
0 & |W|^{2} & 0 \\
0 & 0 & |Z|^{2}|W|^{2}
\end{array}\right) H^{*},
$$

where $H \in S_{\theta}^{3}$ is chosen such that $H H^{*}$ is invertible. Since neither $|Z|^{2}$ nor $|W|^{2}$ is a zero divisor $h$ is clearly non-degenerate, and since $|Z|^{2}$ and $|W|^{2}$ are both central and hermitian it is clear that $h_{a b}=h_{a b}^{*}$ for $a, b=1,2,3$. Thus, $\left(C_{S_{\theta}^{3}}, h\right)$ is a real metric calculus.

We would like a metric and torsion-free affine connection $\nabla$ on $(M, \mathfrak{g})$. From Theorem 2.1.6 it is clear that there can be at most one such connection, and if it exists it can be found using Koszul's formula together with the ansatz $\nabla_{i} E_{j}=$ $E_{1} a_{i j}+E_{2} b_{i j}+E_{3} c_{i j}$ for $i, j=1,2,3$, where $\nabla_{i}=\nabla_{\partial_{i}}$ (note that since $|Z|^{2}$ and $|W|^{2}$ have no inverse in $S_{\theta}^{3}$, the metric $h$ is not invertible, meaning that $\left(C_{S_{\theta}^{3}}, h\right)$ is not a free real metric calculus. Thus, the Levi-Civita connection cannot be guaranteed). Using this method it is possible to explicitly calculate $\nabla_{i} E_{j}$ by first calculating $h\left(\nabla_{i} E_{j}, E_{k}\right)$ using Kozul's formula, and then doing it again using the ansatz. For instance, with $i=j=1$ we get:

$$
\begin{aligned}
& \left\{\begin{array}{l}
h\left(\nabla_{1} E_{1}, E_{1}\right)=\frac{1}{2} \partial_{1} h\left(E_{1}, E_{1}\right)=\frac{1}{2} \partial_{1}\left(H H^{*}\right)|Z|^{2} \\
h\left(\nabla_{1} E_{1}, E_{1}\right)=h\left(E_{1} a^{1,1}, E_{1}\right)=\left(a^{11}\right)^{*} H H^{*}|Z|^{2}
\end{array}\right. \\
& \left\{\begin{array}{l}
h\left(\nabla_{1} E_{1}, E_{2}\right)=-\frac{1}{2} \partial_{2} h\left(E_{1}, E_{1}\right)=-\frac{1}{2} \partial_{2}\left(H H^{*}\right)|Z|^{2} \\
h\left(\nabla_{1} E_{1}, E_{2}\right)=h\left(E_{2} b^{11}, E_{2}\right)=\left(b^{11}\right)^{*} H H^{*}|W|^{2}
\end{array}\right. \\
& \left\{\begin{array}{l}
h\left(\nabla_{1} E_{1}, E_{3}\right)=-\frac{1}{2} \partial_{3} h\left(E_{1}, E_{1}\right)=-\frac{1}{2}\left(\partial_{3}\left(H H^{*}\right)|Z|^{2}+2 H H^{*}|Z|^{2}|W|^{2}\right) \\
h\left(\nabla_{1} E_{1}, E_{3}\right)=h\left(E_{3} c^{11}, E_{3}\right)=\left(c^{11}\right)^{*} H H^{*}|Z|^{2}|W|^{2} .
\end{array}\right.
\end{aligned}
$$


From this calculation it becomes clear that unless the multiplicative inverse of $|W|^{2}$ exists in $S_{\theta}^{3}$ it is not possible to find an expression for $\nabla_{i} E_{j}$ for different values of $i, j$ unless $H$ is chosen so that $\partial_{i}\left(H H^{*}\right)=0$ for $i=1,2,3$ (as was done in AW17b], where the choice $H=\mathbb{1}$ implied the existence of a Levi-Civita connection without the metric $h$ being invertible). The same problem exists for $|Z|^{2}$, and it is solved by introducing inverses $|Z|^{-2},|W|^{-2}$ to $S_{\theta}^{3}$ (this is similar to what is done in AW17a for the noncommutative 4-sphere); the resulting algebra corresponds to a localization of the 3 -sphere, which is why the resulting expanded algebra is denoted by $S_{\theta, \text { loc }}^{3}$.

Extending $M$ to be an $S_{\theta, \text { loc }}^{3}$-module is trivial, and extending the derivations $\partial_{1}, \partial_{2}, \partial_{3}$ so that they can be considered as derivations over $S_{\theta, \text { loc }}^{3}$ is made possible by utilizing the product rule for derivations together with the fact that $0=\partial_{i}(\mathbb{1})=$ $\partial_{i}\left(|Z|^{2}|Z|^{-2}\right)=\partial_{i}\left(|W|^{2}|W|^{-2}\right)$ for $i=1,2,3$; for instance,

$$
0=\partial_{1}(\mathbb{1})=\partial_{1}\left(|Z|^{2}|Z|^{-2}\right)=\partial_{1}\left(|Z|^{2}\right)|Z|^{-2}+|Z|^{2} \partial_{1}\left(|Z|^{-2}\right)=|Z|^{2} \partial_{1}\left(|Z|^{-2}\right),
$$

which implies that $\partial_{1}\left(|Z|^{-2}\right)=0$ since $|Z|^{2}$ is not a zero divisor. Also clear is that $h$ as defined earlier is still a metric on the extended version of $M$, so working on $S_{\theta, \text { loc }}^{3}$ is essentially the same as working on $S_{\theta}^{3}$.

As before, $\left(C_{S_{\theta, \text { loc }}^{3}}, h\right)$ is a real metric calculus over $C_{S_{\theta, \text { loc }}^{3}}$, and since $C_{S_{\theta, \text { loc }}^{3}}$ is a free real calculus and $h$ is invertible, Proposition 2.3.13 now guarantees the existence of a Levi-Civita connection:

Proposition 4.2.3. There exists a unique affine connection $\nabla$ such that $\left(C_{S_{\theta, \text { loc }}^{3}}, h, \nabla\right)$ is a pseudo-Riemannian calculus, with

$$
\begin{aligned}
& \nabla_{1} E_{1}=E_{1} H_{1}-E_{2}|Z|^{2}|W|^{-2} H_{2}-E_{3}\left(|W|^{-2} H_{3}+\mathbb{1}\right) \\
& \nabla_{1} E_{2}=\nabla_{2} E_{1}=E_{1} H_{2}+E_{2} H_{1} \\
& \nabla_{1} E_{3}=\nabla_{3} E_{1}=E_{1}\left(H_{3}+|W|^{2}\right)+E_{3} H_{1} \\
& \nabla_{2} E_{2}=-E_{1}|W|^{2}|Z|^{-2} H_{1}+E_{2} H_{2}+E_{3}\left(\mathbb{1}-|Z|^{-2} H_{3}\right) \\
& \nabla_{2} E_{3}=\nabla_{3} E_{2}=E_{2}\left(H_{3}-|Z|^{2}\right)+E_{3} H_{2} \\
& \nabla_{3} E_{3}=-E_{1}|W|^{2} H_{1}-E_{2}|Z|^{2} H_{2}+E_{3}\left(H_{3}+|W|^{2}-|Z|^{2}\right),
\end{aligned}
$$

where $H_{i}=\frac{1}{2}\left(H H^{*}\right)^{-1} \partial_{i}\left(H H^{*}\right)$ for $i=1,2,3$.

\subsection{An embedding of the noncommutative torus}

We now construct an embedding $(\phi, \psi, \hat{\psi}): C_{S_{\theta, \text { loc }}^{3}} \rightarrow C_{T_{\theta}^{2}}$ by setting

$$
\begin{gathered}
\phi(Z)=\lambda U \\
\phi(W)=\mu V,
\end{gathered}
$$

where $\lambda$ and $\mu$ are complex nonzero (to ensure that $\phi$ is surjective) constants such that $|\lambda|^{2}+|\mu|^{2}=1$ (which implies that $\phi(\mathbb{1})=\mathbb{1}$ ). With these conditions it is 
easy to verify that $\phi$ is a *-algebra epimorphism, and one quickly verifies that the Lie algebra homomorphism $\psi: \mathfrak{g}^{\prime} \rightarrow \mathfrak{g}$ given by

$$
\psi\left(\delta_{1}\right)=\partial_{1} \quad \text { and } \quad \psi\left(\delta_{2}\right)=\partial_{2}
$$

is compatible with $\phi$, giving $M_{\Psi}$ to be the submodule of $M$ generated by $E_{1}$ and $E_{2}$. Finally, with

$$
\hat{\psi}\left(E_{1}\right)=e_{1} \quad \text { and } \quad \hat{\psi}\left(E_{2}\right)=e_{2}
$$

it becomes straightforward to verify that $(\phi, \psi, \hat{\psi}): C_{S_{\theta, \text { loc }}^{3}} \rightarrow C_{T_{\theta}^{2}}$ is a real calculus homomorphism. Furthermore, since $\phi$ is an epimorphism and $M=M_{\Psi} \oplus \tilde{M}$ (where $\tilde{M}$ is the submodule of $M$ generated by $E_{3}$; we note in particular that $\tilde{M}$ is the orthogonal complement of $M_{\Psi}$ with respect to the metric $\left.h\right),(\phi, \psi, \hat{\psi})$ is an embedding of $C_{T_{\theta}^{2}}$ into $C_{S_{\theta, \text { loc }}^{3}}$.

Next, let us calculate the induced metric $h^{\prime}$ on $M^{\prime}$ such that $(\phi, \psi, \hat{\psi})$ is an isometric embedding of $\left(C_{T_{\theta}^{2}}, h^{\prime}\right)$ into $\left(C_{S_{\theta, \text { loc }}^{3}}, h\right)$. Since $M^{\prime}$ has a basis $\left\{e_{1}, e_{2}\right\}$ it is sufficient to calculate $h_{i j}^{\prime}=h^{\prime}\left(e_{i}, e_{j}\right)$ for $i, j=1,2$ :

$$
\begin{aligned}
& h_{11}^{\prime}=\phi\left(h_{11}\right)=\phi\left(H H^{*}|Z|^{2}\right)=|\lambda|^{2} \phi\left(H H^{*}\right) \\
& h_{12}^{\prime}=h_{21}^{\prime}=\phi\left(h_{12}\right)=0 \\
& h_{22}^{\prime}=\phi\left(h_{22}\right)=\phi\left(H H^{*}|W|^{2}\right)=|\mu|^{2} \phi\left(H H^{*}\right) ;
\end{aligned}
$$

it is easy to check that $h^{\prime}$ is an invertible metric on $M^{\prime}$ such that $h_{i j}^{\prime}=\left(h_{i j}^{\prime}\right)^{*}$ for $i, j=1,2$, implying that $\left(C_{T, 2_{\theta}}, h^{\prime}\right)$ is indeed a free real metric caclulus. Moreover, as noted earlier the module $\tilde{M}$ that satisfies $M=M_{\Psi} \oplus \tilde{M}$ is $M_{\Psi}^{\perp}$. Thus, $(\phi, \psi, \hat{\psi})$ is an isometric embedding of $\left(C_{T_{\theta}^{2}}, h^{\prime}\right)$ into $\left(C_{S_{\theta, \text { loc }}^{3}}, h\right)$, as desired.

Since $\left(C_{T_{\theta}^{2}}, h^{\prime}\right)$ is free there is a unique Levi-Civita connection $\nabla^{\prime}$ such that $\left(C_{T_{\theta}^{2}}, h^{\prime}, \nabla^{\prime}\right)$ is pseudo-Riemannian, and $\nabla^{\prime}$ may be retrieved directly from $\nabla$ by using Proposition 3.2 .1 .

$$
\begin{aligned}
& \nabla_{1}^{\prime} e_{1}=\hat{\psi}\left(L\left(\delta_{1}, \Psi\left(\delta_{1}\right)\right)\right)=e_{1} \phi\left(H_{1}\right)-e_{2} \phi\left(H_{2}\right)|\lambda|^{2}|\mu|^{-2} \\
& \nabla_{1}^{\prime} e_{2}=\nabla_{2}^{\prime} e_{1}=\hat{\psi}\left(L\left(\delta_{1}, \Psi\left(\delta_{2}\right)\right)\right)=e_{1} \phi\left(H_{2}\right)+e_{2} \phi\left(H_{1}\right) \\
& \nabla_{2}^{\prime} e_{2}=\hat{\psi}\left(L\left(\delta_{2}, \Psi\left(\delta_{2}\right)\right)\right)=-e_{1} \phi\left(H_{1}\right)|\lambda|^{-2}|\mu|^{2}+e_{2} \phi\left(H_{2}\right),
\end{aligned}
$$

where $H_{i}=\frac{1}{2}\left(H H^{*}\right)^{-1} \partial_{i}\left(H H^{*}\right)$ for $i=1,2,3$ as in Proposition 4.2.3. One also immediately obtains the second fundamental form as

$$
\begin{aligned}
& \alpha\left(\delta_{1}, \Psi\left(\delta_{1}\right)\right)=-E_{3}\left(|W|^{-2} H_{3}+\mathbb{1}\right) \\
& \alpha\left(\delta_{1}, \Psi\left(\delta_{2}\right)\right)=\alpha\left(\delta_{2}, \Psi\left(\delta_{1}\right)\right)=0 \\
& \alpha\left(\delta_{2}, \Psi\left(\delta_{2}\right)\right)=E_{3}\left(\mathbb{1}-|Z|^{-2} H_{3}\right),
\end{aligned}
$$

giving the mean curvature

$$
\begin{aligned}
H_{T_{\theta}^{2}}= & \phi\left(h\left(m, \alpha\left(\delta_{1}, \Psi\left(\delta_{1}\right)\right)\right)\right)\left(h^{\prime}\right)^{11}+\phi\left(h\left(m, \alpha\left(\delta_{2}, \Psi\left(\delta_{2}\right)\right)\right)\right)\left(h^{\prime}\right)^{22} \\
= & \phi\left(h\left(m,-E_{3}\left(|W|^{-2} H_{3}+\mathbb{1}\right)\right)\right)|\lambda|^{-2} \phi\left(H H^{*}\right)^{-1} \\
& +\phi\left(h\left(m, E_{3}\left(\mathbb{1}-|Z|^{-2} H_{3}\right)\right)\right)|\mu|^{-2} \phi\left(H H^{*}\right)^{-1} \\
= & \phi\left(h\left(m, E_{3}\right)\right)\left(|\mu|^{-2}-|\lambda|^{-2}-2|\lambda|^{-2}|\mu|^{-2} \phi\left(H_{3}\right)\right) \phi\left(H H^{*}\right)^{-1} .
\end{aligned}
$$


For the embedding $(\phi, \psi, \hat{\psi}), M_{\Psi}^{\perp}$ is the submodule of $M$ generated by the basis element $E_{3}$. Hence, the mean curvature is zero if

$$
\begin{aligned}
0=H_{T_{\theta}^{2}}\left(E_{3}\right) & =\phi\left(H H^{*}\right)|\lambda|^{2}|\mu|^{2}\left(|\mu|^{-2}-|\lambda|^{-2}-2|\lambda|^{-2}|\mu|^{-2} \phi\left(H_{3}\right)\right) \phi\left(H H^{*}\right)^{-1} \\
& =\phi\left(H H^{*}\right)\left(|\lambda|^{2}-|\mu|^{2}-2 \phi\left(H_{3}\right)\right) \phi\left(H H^{*}\right)^{-1} \\
& =\left(|\lambda|^{2}-|\mu|^{2}\right) \mathbb{1}-2 \phi\left(H H^{*}\right) \phi\left(H_{3}\right) \phi\left(H H^{*}\right)^{-1} \\
& =\left(|\lambda|^{2}-|\mu|^{2}\right) \mathbb{1}-2 \phi\left(H H^{*}\right) \phi\left(\frac{1}{2}\left(H H^{*}\right)^{-1} \partial_{3}\left(H H^{*}\right)\right) \phi\left(H H^{*}\right)^{-1} \\
& =\left(|\lambda|^{2}-|\mu|^{2}\right) \mathbb{1}-\phi\left(\partial_{3}\left(H H^{*}\right)\right) \phi\left(H H^{*}\right)^{-1},
\end{aligned}
$$

implying that the embedding of $\left(C_{T_{\theta}^{2}}, h^{\prime}\right)$ into $\left(C_{S_{\theta}^{3}, \text { loc }}, h\right)$ is minimal if and only if

$$
\phi\left(\partial_{3}\left(H H^{*}\right)\right)=\left(|\lambda|^{2}-|\mu|^{2}\right) \phi\left(H H^{*}\right) .
$$

In the special case where $\phi\left(\partial_{3}\left(H H^{*}\right)\right)=0$, the embedding is minimal if $|\lambda|=$ $|\mu|=1 / \sqrt{2}$ in analogy with the classical case. For the same values of $|\lambda|$ and $|\mu|$ one may also choose, e.g., $H=Z W$ giving $H H^{*}=|Z|^{2}\left|W^{2}\right|$ and

$$
\phi\left(\partial_{3}\left(H H^{*}\right)\right)=2|\lambda|^{2}|\mu|^{2}\left(|\mu|^{2}-|\lambda|^{2}\right)=0 .
$$





\section{5 - Real calculi over matrix algebras}

As a final topic of this thesis we shall discuss matrix algebras. These algebras are well known and understood, and provide an ideal testing ground for developing a deeper intuition as to how real calculi work on a structural level at times where geometric intuition can not be used as a guide like it was for the noncommutative torus and 3-sphere. Matrix algebras are important in noncommutative geometry, not just because they can be used to give rise to interesting examples, but they also have a theoretical significance; for instance, see the spectral Standard Model described in [CC08, where the finite-dimensional algebra $\mathcal{A}_{F}=\mathbb{C} \oplus \mathbb{H} \oplus \operatorname{Mat}_{3}(\mathbb{C})$ encodes the symmetries of the standard model. Therefore, the study of real calculi over matrix algebras is an important part of understanding how real calculi as a framework fit into noncommutative geometry on a larger scale. In this chapter we shall initially describe isomorphisms of real calculi over $\mathcal{A}=\operatorname{Mat}_{N}(\mathbb{C})$, after which we shall resolve the classification problem for real calculi of the form $C_{\mathcal{A}}=\left(\operatorname{Mat}_{N}(\mathbb{C}), \mathfrak{g}_{D}, \mathbb{C}^{N}, \varphi\right)$ when $\operatorname{dim} \mathfrak{g}=1$. We also discuss geometric aspects of this case, and as a final note we discuss briefly how it can be generalized to the case where $C_{\mathcal{A}}=\left(\operatorname{Mat}_{N}(\mathbb{C}), \mathfrak{g}_{D},\left(\mathbb{C}^{N}\right)^{n}, \varphi\right)$ and $\mathfrak{g}$ is an $n$-dimensional abelian Lie algebra.

\subsection{Matrix algebras and classification}

Although the classification problem for general real calculi is complicated, the compatibility conditions between the maps $\phi, \psi$ and $\hat{\psi}$ constituting an isomorphism $(\phi, \psi, \hat{\psi}): C_{\mathcal{A}} \rightarrow C_{\mathcal{A}^{\prime}}$ imply that for a given ${ }^{*}$-isomorphism $\phi: \mathcal{A} \rightarrow \mathcal{A}^{\prime}$ there is at most one choice of $\psi$ and $\hat{\psi}$ such that $(\phi, \psi, \hat{\psi})$ is a real calculus homomorphism (see the discussion in Chapter 2 on real calculus homomorphisms); in general, the existence of a Lie algebra isomorphism $\psi: \mathfrak{g}^{\prime} \rightarrow \mathfrak{g}$ compatible with $\phi$ depends on the representations $D$ and $D^{\prime}$, and the existence of a bijective map $\hat{\psi}: M \rightarrow M^{\prime}$ that is compatible with $\phi$ and $\psi$ then depends on the relationship between the maps $\varphi$ and $\varphi^{\prime}$. Thus, when studying the classification problem for real calculi we may consider real calculi $C_{\mathcal{A}}=\left(\mathcal{A}, \mathfrak{g}_{D}, M, \varphi\right)$ where $\mathcal{A}, \mathfrak{g}$ and $M$ are fixed and $D$ and $\varphi$ are allowed to vary. In this chapter we restrict our attention to real calculi over matrix algebras, i.e., the case when $\mathcal{A}=\operatorname{Mat}_{N}(\mathbb{C})$.

By the Skolem-Noether theorem, all automorphisms of $\operatorname{Mat}_{N}(\mathbb{C})$ are inner, i.e., conjugations by a matrix $U \in \mathrm{GL}_{N}(\mathbb{C})$, and going forward we shall write $\phi_{U}$ to denote conjugation by $U \in \mathrm{GL}_{N}(\mathbb{C})$, i.e., $\phi_{U}(A)=U^{-1} A U$ for $A \in \operatorname{Mat}_{N}(\mathbb{C})$. Also, every derivation on $\operatorname{Mat}_{N}(\mathbb{C})$ is inner, i.e., any derivation $\partial \in \operatorname{Der}(\mathcal{A})$ can be identified with the commutator of a unique trace-free matrix $\hat{D} \in \operatorname{Mat}_{N}(\mathbb{C})$ :

$$
\partial=[\hat{D}, \cdot]
$$


It follows that for any given representation $D: \mathfrak{g} \rightarrow \operatorname{Der}(\mathcal{A})$ there is a unique matrix representation $\hat{D}: \mathfrak{g} \rightarrow \operatorname{Mat}_{N}(\mathbb{C})$ such that $\hat{D}(\partial)$ is trace-free and $D(\partial)=$ $[\hat{D}(\partial), \cdot]$ for every $\partial \in \mathfrak{g}$. We call $\hat{D}$ the matrix representation of $\mathfrak{g}$ associated with $D$, and note that since $D$ is faithful it follows that $\hat{D}$ is also a faithful representation of $\mathfrak{g}$. Moreover, since every derivation $D(\partial)$ is assumed to be hermitian this has the effect that $\hat{D}(\partial)$ is anti-hermitian, implying that $\mathfrak{g}$ is isomorphic to a Lie subalgebra of $\mathfrak{s u}(N)$.

When discussing whether two real calculi $C_{\mathcal{A}}$ and $C_{\mathcal{A}}^{\prime}$ are isomorphic when $\mathcal{A}=$ $\operatorname{Mat}_{N}(\mathbb{C})$ the concept of equivalent matrix representations $\hat{D}$ and $\hat{D}^{\prime}$ becomes too restrictive, and we shall instead introduce the broader concept of quasi-equivalent matrix representations.

Definition 5.1.1. Let $\hat{D}$ and $\hat{D}^{\prime}$ be faithful matrix representations of a Lie algebra $\mathfrak{g}$. Then $\hat{D}$ and $\hat{D}^{\prime}$ are said to be quasi-equivalent if there is a matrix $U \in \mathrm{GL}_{N}(\mathbb{C})$ and a Lie algebra automorphism $\psi \in \operatorname{Aut}(\mathfrak{g})$ such that

$$
\hat{D}^{\prime}(\partial)=\phi_{U}(\hat{D}(\psi(\partial)))
$$

for every $\partial \in \mathfrak{g}$, and $\left(\phi_{U}, \psi\right)$ is said to be a realization of the quasi-equivalence.

The representations $\hat{D}$ and $\hat{D}^{\prime}$ being faithful implies that the automorphism $\psi$ in the above definition is unique since

$$
\phi_{U}(\hat{D}(\psi(\partial)))=\phi_{U}(\hat{D}(\tilde{\psi}(\partial))) \Leftrightarrow \hat{D}(\psi(\partial))=\hat{D}(\tilde{\psi}(\partial)) \Leftrightarrow \psi(\partial)=\tilde{\psi}(\partial)
$$

when $\hat{D}$ is faithful. Therefore, we define $\psi_{U}$ to be the unique automorphism satisfying

$$
\hat{D}^{\prime}(\partial)=\phi_{U}\left(\hat{D}\left(\psi_{U}(\partial)\right)\right)
$$

for every $\partial \in \mathfrak{g}$ whenever such an automorphism exists. In the context of homomorphisms of real calculi over $\mathcal{A}=\operatorname{Mat}_{N}(\mathbb{C}), \phi \in \operatorname{Aut}(\mathcal{A})$ and $\psi \in \operatorname{Aut}(\mathfrak{g})$ are compatible (in the sense of Definition 2.2.1 if and only if $(\phi, \psi)$ is a realization of a quasi-equivalence of the matrix representations $\hat{D}$ and $\hat{D}^{\prime}$ (associated with $D$ and $D^{\prime}$ respectively). This, together with Lemma 2.3.2 from the section on free real calculi, can be used to describe how the structure of a free real calculus $C_{\mathcal{A}}=\left(\mathcal{A}, \mathfrak{g}_{D}, M, \varphi\right)$ is completely determined by the representation $D$ (and its associated matrix representation $\hat{D}$ ) when $\mathcal{A}=\operatorname{Mat}_{N}(\mathbb{C})$ and $\mathfrak{g}$ and $M$ are fixed.

Theorem 5.1.2. Let $C_{\mathcal{A}}=\left(\operatorname{Mat}_{N}(\mathbb{C}), \mathfrak{g}_{D}, M, \varphi\right)$ and $C_{\mathcal{A}}^{\prime}=\left(\operatorname{Mat}_{N}(\mathbb{C}), \mathfrak{g}_{D^{\prime}}, M, \varphi^{\prime}\right)$ be free real calculi. Then $C_{\mathcal{A}} \simeq C_{\mathcal{A}}^{\prime}$ if and only if the matrix representations $\hat{D}$ and $\hat{D}^{\prime}$ (associated with $D$ and $D^{\prime}$, respectively) are quasi-equivalent.

Let $C_{\mathcal{A}}=\left(\operatorname{Mat}_{N}(\mathbb{C}), g_{D}, M, \varphi\right)$ and $C_{\mathcal{A}}^{\prime}=\left(\operatorname{Mat}_{N}(\mathbb{C}), g_{D^{\prime}}, M, \varphi^{\prime}\right)$ be real calculi where $\operatorname{dim} \mathfrak{g}=n$. Since quasi-equivalence between the matrix representations $\hat{D}$ and $\hat{D}^{\prime}$ is a necessary and sufficient condition for the existence of compatible automorphisms $\phi$ and $\psi$, this may be assumed when studying the role that the maps $\varphi$ and $\varphi^{\prime}$ play in distinguishing the structures of $C_{\mathcal{A}}$ and $C_{\mathcal{A}}^{\prime}$ from one another. In the case of free real calculi, Theorem 5.1.2 implies that the relationship between $\varphi$ and $\varphi^{\prime}$ is irrelevant in order to determine whether two real calculi are isomorphic, 
but for general projective real calculi the situation changes. To illustrate this we consider the case when $M=\left(\mathbb{C}^{N}\right)^{n}$, where neither $C_{\mathcal{A}}$ nor $C_{\mathcal{A}}^{\prime}$ are free real calculi when $N>1$. Even in the special case when $n=N>1\left(\right.$ so that $\left(\mathbb{C}^{N}\right)^{n} \simeq \operatorname{Mat}_{N}(\mathbb{C})$ is a free module of rank 1$)$, the real calculus $\left(\operatorname{Mat}_{N}(\mathbb{C}), \mathfrak{g}_{D},\left(\mathbb{C}^{N}\right)^{n}, \varphi\right)$ is still merely projective since $\operatorname{dim} \mathfrak{g}=n>1$ (see Corollary 2.3.4.

\subsection{The 1-dimensional case}

When studying real calculi $C_{\mathcal{A}}=\left(\operatorname{Mat}_{N}(\mathbb{C}), \mathfrak{g}_{D},\left(\mathbb{C}^{N}\right)^{n}, \varphi\right)$ it is natural to begin with the simplest such case, i.e., when $n=\operatorname{dim} \mathfrak{g}=1$. Although this may seem trivial at first glance, a more detailed survey of this case yields a lot of insight into what we can expect to find when the dimension of $\mathfrak{g}$ is higher, especially in relation to metrics and connections. Also, due to the simplistic nature of $\operatorname{Aut}(\mathfrak{g}) \simeq \mathbb{R} \backslash\{0\}$ in the one-dimensional case, it becomes possible to give a comprehensive analysis of the classification problem when the module is $\mathbb{C}^{N}$ (viewed as a right module over $\operatorname{Mat}_{N}(\mathbb{C})$, resulting in vectors being written as row vectors rather than column vectors).

Let $C_{\mathcal{A}}=\left(\operatorname{Mat}_{N}(\mathbb{C}), \mathfrak{g}_{D}, \mathbb{C}^{N}, \varphi\right)$ with $\mathfrak{g}=\langle\partial\rangle$. Since $D(\partial)$ is a hermitian derivation, this implies that $\hat{D}(\partial)$ is skew-hermitian and thus diagonizable, with all eigenvalues being imaginary. In particular, if $\lambda_{1}, \ldots, \lambda_{k}$ are the distinct eigenvalues of $\hat{D}(\partial)$ with respective multiplicities $n_{1}, \ldots, n_{k}$, this implies that there is a matrix $U \in \mathrm{GL}_{N}(\mathbb{C})$ such that

$$
\phi_{U}(\hat{D}(\partial))=\bigoplus_{j=1}^{k} \lambda_{j} \mathbb{1}_{n_{j}} .
$$

Setting $\hat{D}^{\prime}(\partial)=\phi_{U}(\hat{D}(\partial))$ and $\varphi^{\prime}(\partial)=\varphi(\partial) U$, it is straightforward to verify that $C_{\mathcal{A}} \simeq C_{\mathcal{A}}^{\prime}=\left(\operatorname{Mat}_{N}(\mathbb{C}), \mathfrak{g}_{D^{\prime}}, \mathbb{C}^{N}, \varphi^{\prime}\right)$.

More generally, since every $\psi \in \operatorname{Aut}(\mathfrak{g})$ is of the form $\psi(\partial)=\mu \partial$ for some $\mu \in$ $\mathbb{R} \backslash\{0\}$, there is a relatively simple criterion for determining whether two real calculi $\left(\operatorname{Mat}_{N}(\mathbb{C}), \mathfrak{g}_{D},\left(\mathbb{C}^{N}\right)^{n}, \varphi\right)$ and $\left(\operatorname{Mat}_{N}(\mathbb{C}), \mathfrak{g}_{D^{\prime}},\left(\mathbb{C}^{N}\right)^{n}, \varphi^{\prime}\right)$ are isomorphic. We state it as follows:

Proposition 5.2.1. Let

$$
C_{\mathcal{A}}=\left(\operatorname{Mat}_{N}(\mathbb{C}),\langle\partial\rangle_{D}, \mathbb{C}^{N}, \varphi\right) \text { and } C_{\mathcal{A}}^{\prime}=\left(\operatorname{Mat}_{N}(\mathbb{C}),\langle\partial\rangle_{D^{\prime}}, \mathbb{C}^{N}, \varphi^{\prime}\right)
$$

be real calculi. Then $C_{\mathcal{A}} \simeq C_{\mathcal{A}}^{\prime}$ if and only if there is a matrix $U \in \mathrm{GL}_{N}(\mathbb{C})$ and $\mu \in \mathbb{R} \backslash\{0\}$ such that

1. $\hat{D}^{\prime}(\partial)=\mu \phi_{U}(\hat{D}(\partial))$

2. $\varphi^{\prime}(\partial)=\varphi(\partial) U$.

The above proposition has some interesting consequences. If $D$ is given, and $\hat{D}(\partial)$ is a diagonal matrix with eigenvalues sorted in a descending order (with respect to imaginary parts), then the above result can be used to determine the 
number of pairwise nonisomorphic real calculi of the form $\left(\operatorname{Mat}_{N}(\mathbb{C}),\langle\partial\rangle_{D}, \mathbb{C}^{N}, \varphi\right)$. Indeed, Proposition 5.2.1 states that

$$
\left(\operatorname{Mat}_{N}(\mathbb{C}),\langle\partial\rangle_{D}, \mathbb{C}^{N}, \varphi\right) \simeq\left(\operatorname{Mat}_{N}(\mathbb{C}),\langle\partial\rangle_{D}, \mathbb{C}^{N}, \varphi^{\prime}\right)
$$

if and only if there is a matrix $U \in \mathrm{GL}_{N}(\mathbb{C})$ and a constant $\mu \in \mathbb{R} \backslash\{0\}$ such that $\hat{D}(\partial)=\mu \phi_{U}(\hat{D}(\partial))$ and $\varphi^{\prime}(\partial)=\varphi(\partial) U$. Since this implies that $\hat{D}(\partial) / \mu$ is similar to $\hat{D}(\partial)$, and since $\hat{D}(\partial)$ is nonzero, we conclude that $|\mu|=1$, with the special case $\mu=-1$ being possible if and only if $\hat{D}(\partial)$ is similar to $-\hat{D}(\partial)$; in the following, we shall refer to matrices $D$ such that $D$ and $-D$ are similar as anti-selfsimilar.

Furthermore, since $\hat{D}(\partial)$ is diagonal with its eigenvalues sorted in a descending order, i.e.,

$$
\hat{D}(\partial)=\bigoplus_{j=1}^{k} \lambda_{j} \mathbb{1}_{n_{j}},
$$

then the condition $\hat{D}(\partial)=\mu \phi_{U}(\hat{D}(\partial))$ implies that $U$ is of the form

$$
U_{+}=\left(\begin{array}{ccc}
U_{1} & & \\
& \ddots & \\
& & U_{k}
\end{array}\right) \quad \text { or } \quad U_{-}=\left(\begin{array}{ll} 
& U_{1} \\
U_{k} &
\end{array}\right) \text {, }
$$

where $U_{j} \in \mathrm{GL}_{n_{j}}(\mathbb{C})$ for $j=1, \ldots, k$. If $\hat{D}(\partial)$ is not anti-selfsimilar, then $U$ is of the form $U_{+}$and $\mu=1$. But if $\hat{D}(\partial)$ is anti-selfsimilar, then $U$ is either of the form $U_{+}$(corresponding to $\mu=1$ ) or of the form $U_{-}$(corresponding to $\mu=-1$ ). Using these forms of $U$, together with determining all possible vectors of the form $v U$ for a given $v \in \mathbb{C}^{N}$, one arrives at the following.

Corollary 5.2.2. Let $D: \mathfrak{g} \rightarrow \operatorname{Der}\left(\operatorname{Mat}_{N}(\mathbb{C})\right)$ be a faithful representation of $\mathfrak{g}=\langle\partial\rangle$, let $k$ be the number of distinct eigenvalues of $\hat{D}(\partial)$ and let $\left|C_{D}\right|$ denote the number of pairwise nonisomorphic real calculi of the form $\left.\operatorname{Mat}_{N}(\mathbb{C}), \mathfrak{g}_{D}, \mathbb{C}^{N}, \varphi\right)$. Then

1. if $\hat{D}(\partial)$ is not anti-selfsimilar, then $\left|C_{D}\right|=2^{k}-1$,

2. if $\hat{D}(\partial)$ is anti-selfsimilar and $k$ is odd, then $\left|C_{D}\right|=2^{(k-1) / 2}\left(1+2^{(k-1) / 2}\right)-1$,

3. if $\hat{D}(\partial)$ is anti-selfsimilar and $k$ is even, then $\left|C_{D}\right|=2^{k / 2-1}\left(1+2^{k / 2}\right)-1$.

As an illustration of the ideas used to arrive at the above corollary, we shall treat the case where $N=2$ explicitly. In this special case $\hat{D}(\partial)$ is always antiselfsimilar since it is a traceless matrix.

Example 3. Let $\mathcal{A}=\operatorname{Mat}_{2}(\mathbb{C})$, and let

$$
\hat{D}(\partial)=\left(\begin{array}{cc}
i & 0 \\
0 & -i
\end{array}\right)
$$


In this case $\hat{D}(\partial)$ is anti-self similar, and we see that

$$
\hat{D}(\partial)=-(-\hat{D}(\partial))=-1 \cdot \phi_{U_{-}}(\hat{D}(\partial))
$$

for any matrix $U_{-}$of the form

$$
U_{-}=\left(\begin{array}{cc}
0 & \lambda_{1} \\
\lambda_{2} & 0
\end{array}\right)
$$

where $\lambda_{1}, \lambda_{2} \in \mathbb{C} \backslash\{0\}$. Also, $\hat{D}(\partial)=1 \cdot \phi_{U_{+}}(\hat{D}(\partial))$ for any matrix $U_{+}$of the form

$$
U_{+}=\left(\begin{array}{cc}
\lambda_{1} & 0 \\
0 & \lambda_{2}
\end{array}\right)
$$

where $\lambda_{1}, \lambda_{2} \in \mathbb{C} \backslash\{0\}$, and thus Proposition 5.2.1 states that

$$
C_{\mathcal{A}}=\left(\operatorname{Mat}_{2}(\mathbb{C}),\langle\partial\rangle_{D}, \mathbb{C}^{2}, \varphi\right) \simeq C_{\mathcal{A}}^{\prime}=\left(\operatorname{Mat}_{2}(\mathbb{C}),\langle\partial\rangle_{D}, \mathbb{C}^{2}, \varphi^{\prime}\right)
$$

iff $\left(\right.$ with $\varphi(\partial)=(a, b) \in \mathbb{C}^{2}$ and $\left.\varphi^{\prime}(\partial)=\left(a^{\prime}, b^{\prime}\right) \in \mathbb{C}^{2}\right)$

$$
\left(a^{\prime}, b^{\prime}\right)=(a, b) U_{-} \quad \text { or } \quad\left(a^{\prime}, b^{\prime}\right)=(a, b) U_{+},
$$

where the matrices $U_{-}$and $U_{+}$are of the form described earlier. We get two distinct cases:

- If $a \neq 0$ and $b \neq 0$, then $C_{\mathcal{A}} \simeq C_{\mathcal{A}}^{\prime}$ iff $a^{\prime} \neq 0$ and $b^{\prime} \neq 0$.

- Otherwise (in the case when $a b=0) C_{\mathcal{A}} \simeq C_{\mathcal{A}}^{\prime}$ iff $a^{\prime} b^{\prime}=0$.

As a result of the above, there are exactly two nonisomorphic real calculi of the form

$$
\left(\operatorname{Mat}_{2}(\mathbb{C}),\langle\partial\rangle_{D}, \mathbb{C}^{2}, \varphi\right),
$$

where $D$ is the representation given above (in accordance with Corollary 5.2 .2 . case 3$)$.

This, in turn, implies that every real calculus of the form

$$
\left(\operatorname{Mat}_{2}(\mathbb{C}),\langle\partial\rangle_{D^{\prime}}, \mathbb{C}^{2}, \varphi^{\prime}\right),
$$

where $D^{\prime}: \mathfrak{g} \rightarrow \operatorname{Der}\left(\operatorname{Mat}_{2}(\mathbb{C})\right)$ is chosen arbitrarily, is isomorphic to either

$$
C_{\mathcal{A}}^{1}=\left(\operatorname{Mat}_{2}(\mathbb{C}),\langle\partial\rangle_{D}, \mathbb{C}^{2}, \varphi_{1}\right) \quad \text { or } \quad C_{\mathcal{A}}^{2}=\left(\operatorname{Mat}_{2}(\mathbb{C}),\langle\partial\rangle_{D}, \mathbb{C}^{2}, \varphi_{2}\right),
$$

where

$$
\varphi_{1}(\partial)=(1,0) \text { and } \varphi_{2}(\partial)=(1,1)
$$

and $D$ is the same representation as before. This has to do with the fact that for any choice of $D^{\prime}, \hat{D}^{\prime}(\partial)$ is a traceless, nonzero and anti-hermitian matrix, implying that $\hat{D}^{\prime}(\partial)$ has eigenvalues $\lambda_{1}=i \mu$ and $\lambda_{2}=-i \mu$ for some $\mu \in \mathbb{R} \backslash\{0\}$, which in turn implies that $\hat{D}^{\prime}$ and $\hat{D}$ are quasi-equivalent. 
Next, we wish to study real metric calculi and connections. We shall do this by realizing the real calculus $C_{\mathcal{A}}=\left(\operatorname{Mat}_{N}(\mathbb{C}), \mathfrak{g}_{D}, \mathbb{C}^{N}, \varphi\right)$ as a projection of a free real calculus. By Proposition 2.3.10 this amounts to finding a projection $P$ : $\operatorname{Mat}_{N}(\mathbb{C}) \rightarrow \operatorname{Mat}_{N}(\mathbb{C})$ and a map $\tilde{\varphi}: \mathfrak{g} \rightarrow \operatorname{Mat}_{N}(\mathbb{C})$ such that $P\left(\operatorname{Mat}_{N}(\mathbb{C})\right) \simeq \mathbb{C}^{N}$ and such that $\varphi=P \circ \tilde{\varphi}$. We identify vectors $v \in \mathbb{C}^{N}$ with matrices whose first row is $v$ and whose other rows are all zero, i.e.,

$$
\left(x_{1}, \ldots, x_{N}\right) \simeq \theta\left(\left(x_{1}, \ldots, x_{N}\right)\right)=\left(\begin{array}{ccc}
x_{1} & \cdots & x_{N} \\
0 & \cdots & 0 \\
\vdots & \ddots & \vdots \\
0 & \cdots & 0
\end{array}\right)
$$

where the map $\theta: \mathbb{C}^{N} \rightarrow \operatorname{Mat}_{N}(\mathbb{C})$ is a monomorphism, implying that $\mathbb{C}^{N}$ is indeed isomorphic to its image under $\theta$. Let $P: \operatorname{Mat}_{N}(\mathbb{C}) \rightarrow \operatorname{Mat}_{N}(\mathbb{C})$ be the projection such that

$$
P\left(\begin{array}{ccc}
a_{11} & \cdots & a_{1 N} \\
a_{21} & \cdots & a_{2 N} \\
\vdots & \ddots & \vdots \\
a_{N 1} & \cdots & a_{N N}
\end{array}\right)=\left(\begin{array}{ccc}
a_{11} & \cdots & a_{1 N} \\
0 & \cdots & 0 \\
\vdots & \ddots & \vdots \\
0 & \cdots & 0
\end{array}\right)=\theta\left(\left(a_{11}, \ldots, a_{1 N}\right)\right)
$$

making it is clear from the map $\theta$ how matrices in $P\left(\operatorname{Mat}_{N}(\mathbb{C})\right)$ are identified with vectors in $\mathbb{C}^{N}$.

Left is the construction of $\tilde{\varphi}: \mathfrak{g} \rightarrow \operatorname{Mat}_{N}(\mathbb{C})$. Since $v_{0}=\varphi(\partial) \simeq \theta(\varphi(\partial))=$ $P(\tilde{\varphi}(\partial))$, and since $\tilde{\varphi}(\partial)$ is a basis of $\operatorname{Mat}_{N}(\mathbb{C})$, it is clear that $\hat{e}=\tilde{\varphi}(\partial)$ must be an invertible matrix whose first row is $v_{0}$. For the sake of simplicity, we take $\hat{e}$ to be a matrix whose rows are pairwise orthogonal. Then $\tilde{\varphi}$ yields us a free real calculus $\tilde{C}_{\mathcal{A}}$ from which we may realize $C_{\mathcal{A}}$ as a projection using $P$. We note, in particular, that

$$
P(\hat{e})=\hat{e} p
$$

where $p=\frac{1}{\left\|v_{0}\right\|^{2}} v_{0}^{\dagger} v_{0}$ is the matrix that projects vectors in $\mathbb{C}^{N}$ onto $v_{0}=\varphi(\partial)$.

Next, let us show that a general metric on $\mathbb{C}^{N}$ is of a simple form.

Proposition 5.2.3. Let $h$ be a metric on the right $\operatorname{Mat}_{N}(\mathbb{C})$-module $\mathbb{C}^{N}$. Then there exists $x \in \mathbb{R} \backslash\{0\}$ such that

$$
h(u, v)=x \cdot u^{\dagger} v \in \operatorname{Mat}_{N}(\mathbb{C})
$$

Proof. Let $h$ be a metric on $\mathbb{C}^{N}$, and let $e_{1}=(1,0, \ldots, 0) \in \mathbb{C}^{N}$. Set $H=h\left(e_{1}, e_{1}\right)$ and use the identity $v=e_{1} \theta(v)$ (where $\theta$ is the identification between $\mathbb{C}^{N}$ and 
$P\left(\operatorname{Mat}_{N}(\mathbb{C})\right)$ described earlier $)$ to calculate $h(u, v)$ for general vectors in $\mathbb{C}^{N}$ :

$$
\begin{aligned}
h(u, v)= & h\left(e_{1} \theta(u), e_{1} \theta(v)=\theta(u)^{\dagger} h\left(e_{1}, e_{1}\right) \theta(v)=\theta(u)^{\dagger} H \theta(v)\right. \\
= & \left(\begin{array}{cccc}
\bar{u}_{1} & 0 & \cdots & 0 \\
\bar{u}_{2} & 0 & \cdots & 0 \\
\vdots & \vdots & \ddots & \vdots \\
\bar{u}_{N} & 0 & \cdots & 0
\end{array}\right)\left(\begin{array}{cccc}
h_{11} & h_{12} & \cdots & h_{1 N} \\
h_{21} & h_{22} & \cdots & h_{2 N} \\
\vdots & \vdots & \ddots & \vdots \\
h_{N 1} & h_{N 2} & \cdots & h_{N N}
\end{array}\right)\left(\begin{array}{cccc}
v_{1} & v_{2} & \cdots & v_{N} \\
0 & 0 & \cdots & 0 \\
\vdots & \vdots & \ddots & \vdots \\
0 & 0 & \cdots & 0
\end{array}\right) \\
= & \left(\begin{array}{c}
\bar{u}_{1} \\
\bar{u}_{2} \\
\vdots \\
\bar{u}_{N}
\end{array}\right)\left(h_{11}, h_{12}, \cdots, h_{1 N}\right)\left(\begin{array}{cccc}
v_{1} & v_{2} & \cdots & v_{N} \\
0 & 0 & \cdots & 0 \\
\vdots & \vdots & \ddots & \vdots \\
0 & 0 & \cdots & 0
\end{array}\right)=h_{11} \cdot u^{\dagger} v .
\end{aligned}
$$

If $h_{11}$ is zero, then $h(u, v)=0$ for every $u, v \in \mathbb{C}^{N}$. Thus, $h_{11} \in \mathbb{R} \backslash\{0\}$ and the statement follows.

We now move on to affine connections on $\mathbb{C}^{N}$. There are several approaches one can take, and one of the most natural ideas is to use the fact that given a connection $\nabla$, then $\nabla_{\partial}$ is a linear transformation of $\mathbb{C}^{N}$ implying that there is a matrix $X \in \operatorname{Mat}_{N}(\mathbb{C})$ such that $\nabla_{\partial} v=v X$ for every $v \in \mathbb{C}^{N}$. In the case where $\mathfrak{g}$ is 1-dimensional this approach works well, but when trying to generalize to higher dimensions this kind of method becomes impractical. We shall instead use a method based on the ideas presented in Chapter 2 with regards to connections on projective modules. By Proposition 2.3.11 every connection $\nabla$ on $P\left(\operatorname{Mat}_{N}(\mathbb{C})\right)$ can be written as $\nabla=P \circ \tilde{\nabla}$ for a connection $\tilde{\nabla}$ on the free $\operatorname{module}_{\tilde{C}} \operatorname{Mat}_{N}(\mathbb{C})$, and thus we may use the structure of the free calculus $\tilde{C}_{\mathcal{A}}$ to define connections on $\mathbb{C}^{N} \simeq P\left(\operatorname{Mat}_{N}(\mathbb{C})\right)$. On the free module one may define an affine connection in terms of its Christoffel symbol $\tilde{\Gamma} \in \mathcal{A}$ (with respect to the basis $\hat{e}=\tilde{\varphi}(\partial)$ ):

$$
\tilde{\nabla}_{\partial} \hat{e}=\hat{e} \tilde{\Gamma}
$$

and for the connection $\nabla=P \circ \tilde{\nabla}$ on $P\left(\mathcal{A}^{n}\right)$ one obtains (using $P(\hat{e})=\hat{e} p$ )

$$
\nabla_{\partial} P(\hat{e})=P\left(\tilde{\nabla}_{\partial} P(\hat{e})\right)=P\left(\tilde{\nabla}_{\partial} \hat{e} p\right)=P(\hat{e} \tilde{\Gamma}) p+P(\hat{e}) \partial(p)=P(\hat{e})(\tilde{\Gamma} p+\partial(p)),
$$

where we recall that

$$
p=\frac{1}{\left\|v_{0}\right\|^{2}} v_{0}^{\dagger} v_{0}
$$

is the matrix projecting vectors in $\mathbb{C}^{N}$ onto $v_{0}=\varphi(\partial)$. Using this together with the identification between $P(\hat{e})$ and $v_{0}$, a general connection $\nabla$ on $\mathbb{C}^{N}$ can be described by

$$
\nabla_{\partial} v_{0}=v_{0}\left(\lambda \mathbb{1}_{N}+\partial(p)\right)
$$

where $\lambda \in \mathbb{C}$ can be chosen arbitrarily.

At this point we have managed to define a connection on the projective module, and by Proposition 5.2 .3 we know that any metric $h$ on $\mathbb{C}^{N}$ must be $h=h_{x}$ : $(u, v) \mapsto x \cdot u^{\dagger} v$. One easily checks that the pair $\left(C_{\mathcal{A}}, h_{x}\right)$ forms a real metric calculus for any $x \in \mathbb{R} \backslash\{0\}$, and so we may use the above characterization of 
connections on $\mathbb{C}^{N}$ to determine the possible real connection calculi on the form $\left(C_{\mathcal{A}}, h_{x}, \nabla\right)$. In general, the existence of a connection $\nabla$ such that $\left(C_{\mathcal{A}}, h_{x}, \nabla\right)$ is a real connection calculus relies upon $v_{0}=\varphi(\partial)$ being an eigenvector of $\hat{D}(\partial)$, as can be seen in the following.

Proposition 5.2.4. Let $\hat{D}:=\hat{D}(\partial)$ be the matrix representation of $\partial \in \mathfrak{g}$. Then for any $x \in \mathbb{R} \backslash\{0\}$, there exists a connection $\nabla: \mathfrak{g} \times \mathbb{C}^{N} \rightarrow \mathbb{C}^{N}$ such that $\left(C_{\mathcal{A}}, h_{x}, \nabla\right)$ is a real connection calculus if and only if $v_{0}=\varphi(\partial)$ is an eigenvector of $\hat{D}$.

Proof. Assume that $\left(C_{\mathcal{A}}, h_{x}, \nabla\right)$ is a real connection calculus. Using equation (5.1), we have that $\nabla_{\partial} v_{0}=v_{0}\left(\lambda \mathbb{1}_{N}+\partial(p)\right)$ for a $\lambda \in \mathbb{C}$. Thus, we find that the symmetry condition for real connection calculi, $h_{x}\left(v_{0}, \nabla_{\partial} v_{0}\right)=h_{x}\left(v_{0}, \nabla_{\partial} v_{0}\right)^{\dagger}$, is equivalent to

$h_{x}\left(v_{0}, \nabla_{\partial} v_{0}\right)=h_{x}\left(v_{0}, v_{0}\left(\lambda \mathbb{1}_{N}+\partial(p)\right)\right)=x \cdot v_{0}^{\dagger} v_{0}\left(\lambda \mathbb{1}_{N}+\partial(p)\right)=x \cdot\left(\bar{\lambda} \mathbb{1}_{N}+\partial(p)\right) v_{0}^{\dagger} v_{0}$,

since $\partial$ is a hermitian derivation and $p=p^{\dagger}$. Using $v_{0}^{\dagger} v_{0}=\left\|v_{0}\right\|^{2} p$, this is equivalent to

$$
\begin{aligned}
(\lambda-\bar{\lambda}) v_{0}^{\dagger} v_{0} & =\partial(p) v_{0}^{\dagger} v_{0}-v_{0}^{\dagger} v_{0} \partial(p) \\
& =\left\|v_{0}\right\|^{2}([\hat{D}, p] p-p[\hat{D}, p])=\left\|v_{0}\right\|^{2}(\hat{D} p+p \hat{D}-2 p \hat{D} p) .
\end{aligned}
$$

Multiplying by $v_{0}$ from the left, we note that $v_{0}$ is a (left) eigenvector of $\hat{D} p+$ $p \hat{D}-2 p \hat{D} p$ with eigenvalue $(\lambda-\bar{\lambda})$, implying that

$$
(\lambda-\bar{\lambda}) v_{0}=v_{0}(\hat{D} p+p \hat{D}-2 p \hat{D} p)=v_{0} \hat{D}\left(\mathbb{1}_{N}-p\right) ;
$$

multiplying this expression from the right by $v_{0}^{\dagger}$, we find that

$$
(\lambda-\bar{\lambda})\left\|v_{0}\right\|^{2}=v_{0} \hat{D}\left(v_{0}^{\dagger}-v_{0}^{\dagger}\right)=0
$$

which in turn implies that

$$
v_{0} \hat{D}\left(\mathbb{1}_{N}-p\right)=0 .
$$

Hence, $v_{0} \hat{D}$ lies in the kernel of $\left(\mathbb{1}_{N}-p\right)$ which is the same as saying that $v_{0}$ is an eigenvector of $\hat{D}$.

To prove sufficiency, let $v_{0}$ be an eigenvector of $\hat{D}$ with eigenvalue $i \mu$ (where $\mu \in \mathbb{R}$ since $\hat{D}$ is anti-hermitian), and let $\nabla_{\partial} v_{0}=v_{0}\left(\lambda \mathbb{1}_{N}+\partial(p)\right)$ for an arbitrary $\lambda \in \mathbb{C}$. Since $p=\frac{1}{\left\|v_{0}\right\|^{2}} v_{0}^{\dagger} v_{0}$ and $\hat{D}^{\dagger}=-\hat{D}$, we have that

$$
\hat{D} p=-\frac{1}{\left\|v_{0}\right\|^{2}}\left(v_{0} \hat{D}\right)^{\dagger} v_{0}=-\frac{1}{\left\|v_{0}\right\|^{2}}\left(i \mu v_{0}\right)^{\dagger} v_{0}=i \mu p
$$

which implies that $\partial(p)=\hat{D} p-p \hat{D}=i \mu p-i \mu p=0$. Thus, $\nabla_{\partial} v_{0}=\lambda v_{0}$, and the symmetry condition $h_{x}\left(v_{0}, \nabla_{\partial} v_{0}\right)=h_{x}\left(v_{0}, \nabla_{\partial} v_{0}\right)^{\dagger}$ then becomes equivalent to

$$
x \lambda \cdot v_{0}^{\dagger} v_{0}=x \bar{\lambda} \cdot v_{0}^{\dagger} v_{0} .
$$

This is true if and only if $\lambda \in \mathbb{R}$, and since $\nabla$ is a connection for every choice of $\lambda \in \mathbb{C}$ this implies that there is a connection $\nabla$ such that $\left(C_{\mathcal{A}}, h_{x}, \nabla\right)$ is a real connection calculus. 
The key insight that $v_{0}$ is a (left) eigenvector of $\hat{D} p+p \hat{D}-2 p \hat{D} p$ in the above proof is straightforward to generalize to higher-dimensional Lie algebras, which is the main reason why this approach is taken when working with affine connections.

From the proof of Proposition 5.2.4 one may extract some additional information besides the main statement. In particular, if $\left(C_{\mathcal{A}}, h_{x}, \nabla\right)$ is a real connection calculus with $\nabla_{\partial} v_{0}=\lambda v_{0}$, then $\lambda \in \mathbb{R}$. With this it becomes straightforward to state a general result regarding the existence of a Levi-Civita connection given a real metric calculus $\left(C_{\mathcal{A}}, h_{x}\right)$.

Proposition 5.2.5. Let $\left(C_{\mathcal{A}}, h_{x}\right)$ be a real metric calculus, where

$$
C_{\mathcal{A}}=\left(\operatorname{Mat}_{N}(\mathbb{C}),\langle\partial\rangle_{D}, \mathbb{C}^{N}, \varphi\right) .
$$

Then there exists a unique connection $\nabla$ such that $\left(C_{\mathcal{A}}, h_{x}, \nabla\right)$ is pseudo-Riemannian if and only if $v_{0}=\varphi(\partial)$ is an eigenvector of $\hat{D}(\partial)$ with eigenvalue $\lambda_{\partial}$. In this case, the Levi-Civita connection $\nabla$ is given by

$$
\nabla_{\partial} v=\lambda_{\partial} v-v \hat{D}(\partial)
$$

for $v \in \mathbb{C}^{n}$.

\subsection{Abelian Lie algebras}

Before moving forward we shall explain how the module $M=\left(\mathbb{C}^{N}\right)^{n}$ is represented for the sake of clarity when $n>1$. Since we are considering right modules, a vector $v \in\left(\mathbb{C}^{N}\right)^{n}$ is written as a row vector in $\mathbb{C}^{N n}$ (often written as $v=\left(v_{1}, \ldots, v_{n}\right) \in$ $\left(\mathbb{C}^{N}\right)^{n}$, with each $\left.v_{i} \in \mathbb{C}^{N}\right)$, with matrices $A \in \operatorname{Mat}_{N}(\mathbb{C})$ acting on $\left(\mathbb{C}^{N}\right)^{n}$ in the following way:

$$
v \cdot A=\left(v_{1}, \ldots, v_{n}\right) \cdot A=\left(v_{1} A, \ldots, v_{n} A\right)=v\left(\begin{array}{lll}
A & & \\
& \ddots & \\
& & A
\end{array}\right)=v\left(\bigoplus_{1}^{n} A\right) .
$$

In general, the dot notation above is used to distinguish the module action from a regular matrix multiplication when $n>1$. Let $C_{\mathcal{A}}=\left(\operatorname{Mat}_{N}(\mathbb{C}), \mathfrak{g}_{D},\left(\mathbb{C}^{N}\right)^{n}, \varphi\right)$ and assume that $\mathfrak{g}$ is abelian with a basis $\left\{\partial_{1}, \ldots, \partial_{n}\right\}$. Some of the results from the previous section can be generalized to higher dimensions without great effort, and to this end we will consider the special case where each $e_{i}=\varphi\left(\partial_{i}\right)$ is of the following form:

$$
e_{i}=\left(0, \ldots, 0, \alpha_{i} v_{0}, 0, \ldots, 0\right) \in\left(\mathbb{C}^{N}\right)^{n},
$$

where $v_{0} \in \mathbb{C}^{N}$ is a unit vector and $\alpha_{i} \in \mathbb{R} \backslash\{0\}$. The effect this has is that for any metric $h$ on $\left(\mathbb{C}^{N}\right)^{n}$ we have that $h\left(e_{i}, e_{j}\right)=\mu_{i j} \alpha_{i} \alpha_{j} v_{0}^{\dagger} v_{0}=\tilde{\mu}_{i j} v_{0}^{\dagger} v_{0}$ where $\mu_{i j} \in \mathbb{R}$ (implying that $\tilde{\mu}_{i j} \in \mathbb{R}$ as well); this can be most easily seen by noting that $h$ can be broken down as the sum of general bilinear forms over $\mathbb{C}^{N}$, after which we can apply the exact same reasoning used to prove Proposition 5.2 .3 to reach this conclusion. Moreover, the symmetry condition $h\left(\varphi\left(\partial_{i}\right), \varphi\left(\partial_{j}\right)\right)=h\left(\varphi\left(\partial_{j}\right), \varphi\left(\partial_{i}\right)\right)$ 
implies that $\tilde{\mu}_{i j}=\tilde{\mu}_{j i} \in \mathbb{R}$ for every pair $i, j$ and the metric $h$ being nondegenerate is reflected in the fact that the real and symmetric $n \times n$ matrix $\tilde{M}=\left(\tilde{\mu}_{i j}\right)$ defining the metric $h$ is invertible.

To realize $\left(C_{\mathcal{A}}, h\right)$ as a projection of a free real metric calculus we need to determine a suitable projection $P: \mathcal{A}^{n} \rightarrow \mathcal{A}^{n}$ and invertible metric $\tilde{h}$ on $\mathcal{A}^{n}$, and to this end we let $A$ be a unitary matrix whose first row is equal to $v_{0}$. We then set $A_{i}=\alpha_{i} A$ and let $\left\{\hat{e}_{i}\right\}_{1}^{n}$ be the basis of $\mathcal{A}^{n}$ given by

$$
\hat{e}_{i}=\left(0, \ldots, 0, A_{i}, 0, \ldots, 0\right) \in \mathcal{A}^{n} .
$$

As a projection we use the the map $P\left(B_{1}, \ldots, B_{n}\right)=\left(\tilde{P}\left(B_{1}\right), \ldots, \tilde{P}\left(B_{n}\right)\right)$, where

$$
\tilde{P}\left(\begin{array}{ccc}
b_{11} & \cdots & b_{1 N} \\
b_{21} & \cdots & b_{2 N} \\
\vdots & \ddots & \vdots \\
b_{N 1} & \cdots & b_{N N}
\end{array}\right)=\left(\begin{array}{ccc}
b_{11} & \cdots & b_{1 N} \\
0 & \cdots & 0 \\
\vdots & \ddots & \vdots \\
0 & \cdots & 0
\end{array}\right)
$$

in analogy with the 1-dimensional case we may define $P$ in terms of the basis $\hat{e}_{i}$ for $i=1, \ldots, n$, obtaining

where the coefficients

$$
P\left(\hat{e}_{j}\right)=\hat{e}_{k} \cdot p_{j}^{k}
$$

$$
p_{j}^{k}= \begin{cases}v_{0}^{\dagger} v_{0}=p & \text { if } j=k \\ 0 & \text { otherwise }\end{cases}
$$

Note that $p$ does not need to be scaled by the factor $\left\|v_{0}\right\|^{2}$, since $v_{0}$ is a unit vector. Identifying $\left(\mathbb{C}^{N}\right)^{n}$ with $P\left(\mathcal{A}^{n}\right)$, we note that $\varphi\left(\partial_{i}\right)=e_{i} \simeq P\left(\hat{e}_{i}\right)$ for $i=1, \ldots, n$, and thus we define the map $\tilde{\varphi}: \mathfrak{g} \rightarrow \mathcal{A}^{n}$ by $\tilde{\varphi}\left(\partial_{i}\right)=\hat{e}_{i}$ for $i=1, \ldots, n$. This yields the free real calculus $\tilde{C}_{\mathcal{A}}=\left(\operatorname{Mat}_{N}(\mathbb{C}), \mathfrak{g}_{D},\left(\operatorname{Mat}_{N}(\mathbb{C})\right)^{n}, \tilde{\varphi}\right)$ from which $C_{\mathcal{A}}$ can be realized as a projection.

Next, we choose the metric $\tilde{h}$ to be given by

$$
\tilde{h}\left(\hat{e}_{i}, \hat{e}_{j}\right)=\tilde{h}_{i j}=\tilde{\mu}_{i j} A^{\dagger} A=\tilde{\mu}_{i j} \mathbb{1}_{N} .
$$

One quickly verifies that $\tilde{h}_{i j}=\tilde{h}_{j i}$ and that $\tilde{h}$ is invertible by calculating $\tilde{h}^{i j} \tilde{h}_{j k}$, where $\tilde{h}^{i j}=\tilde{\mu}^{i j} \mathbb{1}_{N}$. It is straightforward to verify that $P$ is orthogonal with respect to $\tilde{h}$ and that $h\left(e_{i}, e_{j}\right)=\tilde{h}\left(P\left(\hat{e}_{i}\right), P\left(\hat{e}_{j}\right)\right)$, which implies that $\left(C_{\mathcal{A}}, h\right)$ can be realized as the projection of the free real metric calculus $\left(\tilde{C}_{\mathcal{A}}, \tilde{h}\right)$.

We are interested in affine connections $\nabla$ on $\left(\mathbb{C}^{N}\right)^{n}$, and since every such connection is the projection of a connection $\tilde{\nabla}$ on the free module, we may use the structure on the free module to perform calculations. On the free module, it is straightforward to define an affine connection $\tilde{\nabla}$ in terms of its Christoffel symbols $\tilde{\Gamma}_{i j}^{k}$, where

$$
\nabla_{i} \hat{e}_{j}=\hat{e}_{k} \tilde{\Gamma}_{i j}^{k}
$$

Using this, one may calculate corresponding Christoffel symbols $\Gamma_{i j}^{k}$ for the connection $\nabla=P \circ \tilde{\nabla}$ :

$$
\begin{aligned}
\nabla_{i} P\left(\hat{e}_{j}\right) & =P\left(\tilde{\nabla}_{i} P\left(\hat{e}_{j}\right)\right)=P\left(\tilde{\nabla}_{i} \hat{e}_{k} \cdot p_{j}^{k}\right)=P\left(\tilde{\nabla}_{i} \hat{e}_{j} \cdot p\right)=P\left(\hat{e}_{k} \cdot \tilde{\Gamma}_{i j}^{k} p+\hat{e}_{j} \cdot \partial_{i}(p)\right) \\
& =P\left(\hat{e}_{k}\right) \cdot \tilde{\Gamma}_{i j}^{k} p+P\left(\hat{e}_{j}\right) \cdot \partial_{i}(p)=P\left(\hat{e}_{k}\right) \cdot\left(\tilde{\Gamma}_{i j}^{k} p+\delta_{j}^{k} \partial_{i}(p)\right),
\end{aligned}
$$


where $p=v_{0}^{\dagger} v_{0}$ is the matrix projecting vectors in $\mathbb{C}^{N}$ onto $v_{0}$; since we are only interested in connections such that $\left(C_{\mathcal{A}}, h, \nabla\right)$ is a real connection calculus, an analogous line of reasoning to that in Proposition 5.2.4 implies that $v_{0}$ is an eigenvector of $\hat{D}\left(\partial_{i}\right)$ for $i=1, \ldots, n$. Since $\mathfrak{g}$ is abelian $v_{0}$ can be assumed to be such a vector in what follows, implying that $\partial_{i}(p)=0$ for $i=1, \ldots, n$. Thus, using the identification between $e_{i}$ and $P\left(\hat{e}_{i}\right)$, one finds that any connection $\nabla$ of interest can be written as

$$
\nabla_{i} e_{j}=e_{k} \cdot \tilde{\Gamma}_{i j}^{k} p
$$

where $\tilde{\Gamma}_{i j}^{k} \in \operatorname{Mat}_{N}(\mathbb{C})$.

This description of $\nabla$ can be simplified further. By setting $v_{i j}^{k}=v_{0}\left(\tilde{\Gamma}_{i j}^{k}\right)^{\dagger} \in \mathbb{C}^{N}$, one may use the standard scalar product $\langle\cdot, \cdot\rangle$ on $\mathbb{C}^{N}$ to calculate the expression for $\nabla$ :

$e_{k} \cdot \tilde{\Gamma}_{i j}^{k} p=\left(0, \ldots, \alpha_{k} v_{0}, 0, \ldots, 0\right) \cdot\left(v_{i j}^{k}\right)^{\dagger} v_{0}=\left(0, \ldots, \alpha_{k}\left\langle v_{0}, v_{i j}^{k}\right\rangle v_{0}, 0, \ldots, 0\right)=e_{k}\left\langle v_{0}, v_{i j}^{k}\right\rangle$, and if we let $\lambda_{i j}^{k}=\left\langle v_{0}, v_{i j}^{k}\right\rangle \in \mathbb{C}$ we have that

$$
\nabla_{i} e_{j}=e_{k} \lambda_{i j}^{k}
$$

defines a connection on $\left(\mathbb{C}^{N}\right)^{n}$ for arbitrary $\lambda_{i j}^{k} \in \mathbb{C}$.

With this simplification, the hermiticity condition for $h\left(e_{i}, \nabla_{j} e_{k}\right)$ becomes equivalent to stating that $\lambda_{j k}^{l} \in \mathbb{R}$ for every $j, k, l$. Since the torsion condition is not trivial if $\operatorname{dim} \mathfrak{g}>1$, Koszul's formula may be used to find constants $\lambda_{i j}^{k}$ satisfying the metric and torsion-free conditions for $\nabla$. This implies that $h\left(\nabla_{i} e_{j}, e_{k}\right)=0$ for the Levi-Civita connection $\nabla$, since the Lie bracket is zero and $\partial_{i}\left(h_{j k}\right)=0$ for every $i, j, k$. On the other hand, $h\left(\nabla_{i} e_{j}, e_{k}\right)$ may be computed explicitly, and by solving the equation $h\left(\nabla_{i} e_{j}, e_{k}\right)=0$ one finds that $\bar{\lambda}_{i j}^{k}=\lambda_{i j}^{k}=0$ for every $i, j, k$. In other words, for an arbitrary vector $v=e_{k} \cdot B^{k} \in\left(\mathbb{C}^{N}\right)^{n}$ and $\partial \in \mathfrak{g}$,

$$
\begin{aligned}
\nabla_{\partial} v & =\nabla_{\partial}\left(e_{k} \cdot B^{k}\right)=e_{k} \cdot \partial\left(B^{k}\right)=e_{k} \cdot\left[\hat{D}(\partial), B^{k}\right]=\lambda_{\partial} e_{k} \cdot B^{k}-\left(e_{k} \cdot B^{k}\right) \cdot \hat{D}(\partial) \\
& =\lambda_{\partial} v-v \cdot \hat{D}(\partial),
\end{aligned}
$$

where $\nabla$ is the Levi-Civita connection and $\lambda_{\partial}$ is the eigenvalue of $\hat{D}(\partial)$ associated with $v_{0}$. We summarize this discussion as follows.

Proposition 5.3.1. Let $\mathfrak{g}$ be an abelian Lie algebra with basis $\left\{\partial_{1}, \ldots, \partial_{n}\right\}$ and let $\left(C_{\mathcal{A}}, h\right)$ be a real metric calculus such that

$$
C_{\mathcal{A}}=\left(\operatorname{Mat}_{N}(\mathbb{C}), \mathfrak{g}_{D},\left(\mathbb{C}^{N}\right)^{n}, \varphi\right)
$$

and $\varphi\left(\partial_{i}\right)=\left(0, \ldots, 0, \alpha_{i} v_{0}, 0, \ldots, 0\right) \in\left(\mathbb{C}^{N}\right)^{n}$, where $\alpha_{i} \in \mathbb{R} \backslash\{0\}$ and $v_{0}$ is a unit vector. Then there exists a unique connection $\nabla$ such that $\left(C_{\mathcal{A}}, h, \nabla\right)$ is pseudoRiemannian if and only if $v_{0}$ is an eigenvector of $\hat{D}\left(\partial_{i}\right)$ with eigenvalue $\lambda_{i}$ for $i=1, \ldots, n$. In this case, the Levi-Civita connection $\nabla$ is given by

$$
\nabla_{\partial_{i}} v=\lambda_{i} v-v \cdot \hat{D}\left(\partial_{i}\right),
$$

for $v \in\left(\mathbb{C}^{N}\right)^{n}$. 



\section{6 - Outlook}

The concept of real calculus homomorphisms enables a deeper understanding of real calculi on a structural level, and the role that the framework plays in the larger context of noncommutative geometry. While the subjects discussed in this text are certainly important, the current state of the theory should be seen mainly as a starting point. There are many questions left to answer with regards to real calculus homomorphisms, both when interpreted geometrically as well as when they are used to study real calculi as algebraic structures. This is true for the concrete matters discussed in this thesis as well.

For instance, the notion of mean curvature of an embedding developed in this thesis is only applicable for free real metric calculi, which is admittedly a rather severe restriction although it is sufficient when discussing the noncommutative torus and 3-sphere. A natural next step is to define the mean curvature without a need to refer to a specific basis of the Lie algebra in order to have a notion of mean curvature for general projective real calculi.

With regards to matrix algebras, a natural generalization of the scenario described in Section 5.3 is to consider nonabelian Lie algebras $\mathfrak{g}$ and generators $\varphi\left(\partial_{i}\right)=\left(0, \ldots, 0, \tilde{v}_{i}, 0, \ldots, 0\right)$, where the vectors $\tilde{v}_{i} \in \mathbb{C}^{N}$ are not all assumed to be parallell. Many of the ideas with regards to real connection calculi may be transferred to this more general setup, and it is interesting to see whether there is a good way to address the classification problem in this case as well.

Although the definitions and concepts outlined can be discussed further in isolation, it is also interesting to study how the ideas and concepts presented here relate to other approaches to noncommutative geometry. For instance, the work on sigma-model solitons on noncommutative spaces presented in DKL00, DKL03, Lan06, DLL15] is interesting to compare to embeddings as discussed using real calculus homomorphisms. These kinds of comparisons become increasingly important as real calculi grow more robust as a framework in noncommutative geometry.

\section{Bibliography}

[AL20] J. Arnlind and G. Landi. Projections, modules and connections for the noncommutative cylinder. Adv. Theor. Math. Phys., 24(3):527$562,2020$.

[ATN21] J. Arnlind and A. Tiger Norkvist. Noncommutative minimal embeddings and morphisms of pseudo-riemannian calculi. J. Geom. Phys., $159(1), 2021$. 
[AW17a] J. Arnlind and M. Wilson. On the Chern-Gauss-Bonnet theorem for the noncommutative 4-sphere. J. Geom. Phys., 111:126-141, 2017.

[AW17b] J. Arnlind and M. Wilson. Riemannian curvature of the noncommutative 3-sphere. J. Noncommut. Geom., 11(2):507-536, 2017.

[BM11] E. J. Beggs and S. Majid. *-compatible connections in noncommutative Riemannian geometry. J. Geom. Phys., 61(1):95-124, 2011.

[CC08] A.H. Chamseddine and A. Connes. Why the standard model. J. Geom. Phys., 58:38-47, 2008.

[Con85] Alain Connes. Non-commutative differential geometry. Publications Mathématiques de l'IHÉS, 62:41-144, 1985.

[Con94] A. Connes. Noncommutative geometry. Academic Press, Inc., San Diego, CA, 1994.

[DKL00] L. Dabrowski, T. Krajewski, and G. Landi. Some properties of nonlinear $\sigma$-models in noncommutative geometry. Int. J. Mod. Phys. B, 14:2367-2382, 2000.

[DKL03] L. Dabrowski, T. Krajewski, and G. Landi. Non-linear $\sigma$-models in noncommutative geometry:fields with values in finite spaces. Mod. Phys. Lett. A, 18:2367-2382, 2003.

[DLL15] L. Dabrowski, G. Landi, and F. Luef. Sigma-model solitons on noncommutative spaces. Lett. Math. Phys. A, 105:1663--1688, 2015.

[DV88] M. Dubois-Violette. Dérivations et calcul différentiel non commutatif. C. R. Acad. Sci. Paris Sér. I Math., 307(8):403-408, 1988.

[DVKM90] M. Dubois-Violette, R. Kerner, and J. Madore. Noncommutative differential geometry of matrix algebras. J. Math. Phys., 31:316, 1990.

[FGR99] J. Fröhlich, O. Grandjean, and A. Recknagel. Supersymmetric quantum theory and non-commutative geometry. Comm. Math. Phys., 203(1):119-184, 1999.

[Lan06] G. Landi. On harmonic maps in noncommutative geometry. In Noncommutative Geometry and Number Theory: Where Arithmetic meets Geometry and Physics, pages 217-234. Vieweg, Wiesbaden, 2006.

[LM87] G. Landi and G. Marmo. Lie algebra extensions and abelian monopoles. Phys. Lett. B, 195(3):429-434, 1987.

[Ros13] J. Rosenberg. Levi-Civita's theorem for noncommutative tori. SIGMA, 9:071, 2013.

[SW99] Nathan Seiberg and Edward Witten. String theory and noncommutative geometry. Journal of High Energy Physics, 1999(09):032, 1999.

[TN21] A. Tiger Norkvist. Real calculi over finite noncommutative spaces. preprint, 2021. 


\section{Papers}

The papers associated with this thesis have been removed for copyright reasons. For more details about these see:

http://urn.kb.se/resolve?urn=urn:nbn:se:liu:diva-175740 


\section{FACULTY OF SCIENCE AND ENGINEERING}

Linköping Studies in Science and Technology. Licentiate Thesis No. 1913, 2021 Department of Mathematics

Linköping University

SE-581 83 Linköping, Sweden

www.liu.se 